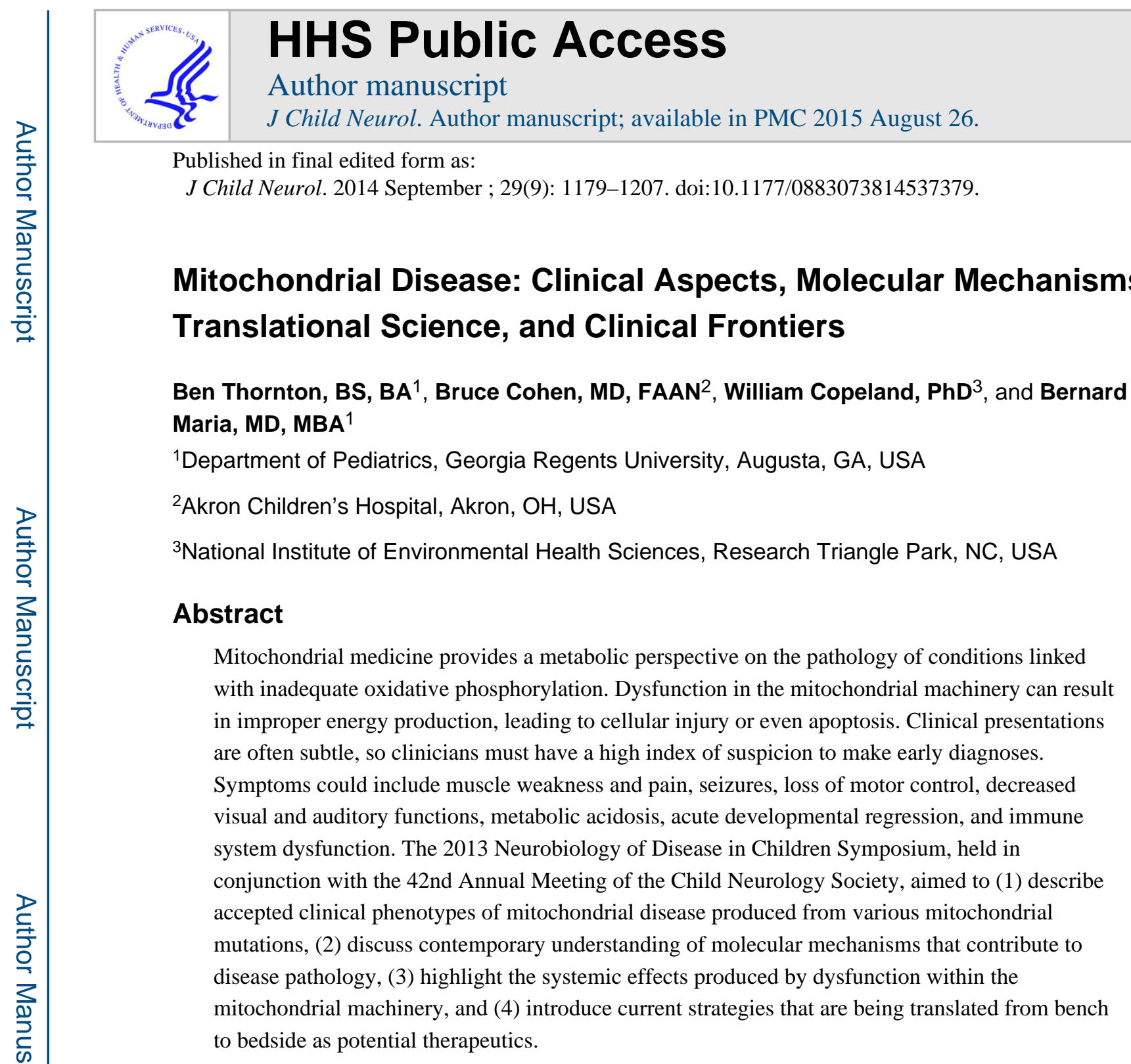

\title{
Keywords
}

mitochondrial disease

\section{Clinical Aspects}

Moderator: Bruce Cohen, MD, Akron Children's Hospital, Akron, Ohio

\section{Natural History and Classification of Mitochondrial Disease}

Eric Schon, PhD, Columbia University, New York, New York

\footnotetext{
Reprints and permission: sagepub.com/journalsPermissions.nav

Corresponding Author: Bernard L. Maria, MD, MBA, Department of Pediatrics, Medical College of Georgia at Georgia Regents University, 1446 Harper Street, BT-1850, Augusta, GA 30912, USA. bmaria@gru.edu.

Declaration of Conflicting Interests

The authors declared no potential conflicts of interest with respect to the research, authorship, and/or publication of this article.
} 
Dr Schon opened the conference by providing an overview of the mitochondrial organelle, its housekeeping and specialized functions, and its contributions to the current understanding of mitochondrial disease. An individual cell uses 2 kinds of genetic material -nuclear DNA and mitochondrial DNA - to maintain standard cellular function. As a prehistoric prokaryote, the mitochondrial organelle has over the years created an endosymbiotic relationship with its eukaryotic host to provide cellular energy in exchange for gaining nuclear benefits as an essential component of the cellular structure. During eukaryotic evolution, mitochondria became invaginated by the eukaryotic host, resulting in the development of a double membrane that enables the production of energy via oxidative phosphorylation. In addition, the mitochondrion contains genes and gene products that help maintain the cell's shape, produce locomotion, and imports components from its outside environment via transporter proteins. Mitochondria also can signal other components of the cell in order to complete a multitude of tasks. The mitochondrion imports as many as 2000 gene products from the nuclear genome to function properly.

Dr Schon briefly reviewed the importance of targeting sequences in the mitochondrial importation process. The targeting sequence, also known as the presequence, is imported into the mitochondria and then binds to the apical portion of the inner membrane. As it is processed at the apical surface, the presequence is threaded into the matrix, where it is translated into a primary structure of amino acids; then, the presequence is disposed of, and the resulting protein structure is reassembled in the interior matrix of the mitochondria. This conserved process is crucial to the viability of the mitochondria; therefore, any disruption of the process is associated with dysfunction and diseases.

Most mitochondrial diseases arise from a disruption in oxidative phosphorylation. When this process is impeded, the mitochondria's essential role to produce energy in the form of adenosine triphosphate (ATP) is impaired, resulting in oxidative cellular injury and cell death. A critical component of the oxidative phosphorylation mechanism is the respiratory chain located on the inner mitochondrial matrix. The respiratory chain is considered a bioenergetics composite consisting of 5 complexes (complexes I, II, III, IV, and V). Each complex consists of various subunits encoded by nuclear DNA, which is imported into the mitochondria. The gene products, in a process that is poorly understood, then combine to comprise the inner mitochondrial respiratory chain. Electrons are transported horizontally along the axis of the inner mitochondrial respiratory chain while simultaneously pumping protons into the inner membrane space, resulting in an electrochemical gradient. This situation is analogous to a biological compositor, which uses potential energy in the form of a charge distribution across the inner mitochondrial respiratory chain to drive ATP production via an adenosine triphosphatase (ATPase) located in a hexameric protein structure in complex V. Because of the hexameric structure of complex V, the protein can produce 3 ATP units per cycle, at a rate of 1000 rotations per minute.

Mitochondrial genetics are transmitted in a Mendelian fashion but via uniparental inheritance. In addition, mitochondrial segregation during germline development follows a bottleneck sampling effect. Prior to the formation of a zygote, only a small fraction of the mother's mitochondrial DNA is shared with progeny. This limits the probability that a mutation will be transferred to the next generation. In addition to the first bottleneck 
sampling effect, there is also a somatic bottleneck event. As the zygote progresses to the multicellular stage (up to 128 cells), only approximately 3 of those cells will contribute to the formation of a newborn child. The other cells will be allocated to support the developing fetus as extra-embryonic tissue. This sampling effect can partially explain why different patients have varying severities of disease, depending on how much mutated mitochondrial DNA a child inherits from his or her mother.

Lactic acidosis is caused by pyruvate accumulation when the inner mitochondrial respiratory chain is dysfunctional. The morphology of this occurrence is seen as ragged red fibers in muscle pathologic specimens. As mentioned earlier, the mitochondria use genetic products from mitochondrial DNA as well as from nuclear DNA. Complex II, also labeled succinate dehydrogenase, is composed exclusively of nuclear components. The ragged red appearance is a classic morphologic feature of mitochondrial disease caused by high levels of succinate dehydrogenase generated to compensate for low levels of oxygen.

It is important to have a firm understanding of mitochondrial genetics to understand mitochondrial disease and its clinical presentation. Human tissues as a rule contain multiple mitochondrial genomes; therefore, if there is a mutation in one of them, it will be compensated for by the other nonmutated mitochondrial genomes. This compensatory effect lends itself to the concept of a threshold effect, in which the body can tolerate up to a certain level of mitochondrial dysfunction. Thus, diseases manifest clinically when mutated mitochondrial DNA exceed a certain threshold.

Mitochondrial encephalomyopathy, lactic acidosis, and strokelike episodes, or MELAS, is a prototypical disorder that exemplifies the threshold effect. This disease is maternally inherited and is caused by an A to G point mutation in a tRNA at nt 3243 that encodes for leucine. In a patient described by Dr Schon, a muscle biopsy showed heteroplasmy, in which the tissue contained $80 \%$ mutated mitochondrial DNA, above the tolerated threshold to cause disease. The MELAS patient inherited the mutation from the mother, not only in muscle tissue but also in the liver, kidney, heart, and brain, illustrating the point that mitochondrial disease can affect multiple organ systems and tissues. MELAS patients typically have muscle biopsies revealing classic COX-positive ragged red fibers.

Kearns-Sayre syndrome is a fatal disorder, with onset before 20 years of age, caused by a large-scale deletion (most commonly a 4977 base pair deletion) in mitochondrial genome. Clinical presentations include ophthalmoplegia and ptosis, pigmentary retinopathy, heart conduction block, cerebellar ataxia, hearing loss, and diabetes. In comparison with patients who have MELAS, patients with Kearns-Sayre syndrome also present with ragged-red fibers that are COX-negative because their mitochondrial DNA genome is not synthesizing COX 2 , an important subunit of the mitochondrial genome.

Dr Schon then discussed new frontiers in mitochondrial research. New research is testing mutations in mitochondria that affect organelle shape, fusion, and division. Mitochondrial quality control, a new topic with Parkinson disease, describes the cell's inability to undergo adequate mitophagy to clear the cell of malfunctioning mitochondria. Communication between mitochondria and other organelles has been offered as a focal point in disease 
pathology. Novel research is directing its attention at the mitochondria's inability to communicate with the endoplasmic reticulum, resulting in diseases such as Alzheimer and juvenile amyotrophic lateral sclerosis.

Mitochondrial dysfunction encompasses a broad class of diseases with varying etiologies and ages of onset. All cells within the body, with the exception of red blood cells, use mitochondria to provide essential energy for cellular growth and development. Depending on the location of the tissue, mitochondria serve varying roles according to the biogenetic demands of the cell. They mutate rapidly as a population, but contain excellent fidelity of replication. Mitochondria play an essential role within the cell; therefore, improper mitochondrial machinery can have dire consequences and results in mitochondrial disorders. Ultimately, understanding the mitochondria's role in the cell and discerning clinical presentation can help guide rational diagnostic testing and future therapeutics.

\title{
Diagnosis and Treatment of Mitochondrial Diseases
}

\author{
Bruce Cohen, MD, Akron Children's Hospital, Akron, Ohio
}

Diagnosing mitochondrial disease using a concrete list of clinical phenotypes is challenging because of the variability in mutations and the process of heteroplasmy described in the previous section. Therefore, the clinician must complete a thorough examination, looking for objective and subjective historical elements of disease. It is also important to use previous studies to see whether there is concordance between the research findings and the clinical presentation of the patient, to rule out any other explanations of the illness.

Mitochondrial disease is characterized by varying patterns and clinical presentations. The classic mitochondrial phenotype is associated with a wide array of clinical symptoms, such as progressive optic atrophy, retinitis pigmentosa, cardiomyopathy, systemic lipomatosis, and large-fiber neuropathy. Classic magnetic resonance imaging (MRI) findings in patients suffering from Leigh syndrome include symmetric, bilateral deep gray matter that originates in the brain stem and spreads to the upper portion of the cerebrum. Despite many years of clinical research, leading experts still disagree about how best to describe the clinical phenotype.

Dr Cohen introduced the concept of levels of certainty, to circumvent any doubts or questions about establishing definitive mitochondrial disease. The clinician can conduct various diagnostic evaluations such as physical examinations, muscle biopsies, and various biochemical tests, then apply points to various diagnostic criteria to provide a "degree of certainty" that the patient has conclusive mitochondrial disease. However, patients might be too intensively studied and because of limited laboratory resources or patient and family preference, multiple clinical evaluations might not be possible. Single clinical evaluations can, in most situations, provide sufficient indication of primary mitochondrial disease, secondary mitochondrial disease, possible mitochondrial disorders, and echogenic mitochondrial disorders.

Primary mitochondrial diseases are illnesses with a classic mitochondrial phenotype. Many primary mitochondrial diseases are caused by pathogenic mutations within the 
mitochondrial DNA or nuclear DNA. Generally, primary mitochondrial diseases are inherited in a recessive manner from asymptomatic parents.

Secondary mitochondrial diseases also are characterized by a classic mitochondrial phenotype; however, although the mitochondrial machinery can be injured by external factors such as toxins, injury to the mitochondrial DNA is not necessary to produce disease. For example, an excessive accumulation of sulfide due to a mutation in mitochondrial deoxygenate enzyme can initiate development of a secondary mitochondrial disease.

In the milieu of mitochondrial illnesses, some clinical presentations mimic mitochondrial disease. These disorders frequently have a classic or less-than-classic phenotype; however, they do not meet the criteria of either primary or secondary mitochondrial disease because the phenotypic appearance is better explained by other pathologies.

The fourth category, mitochondrial ecovariants, develops with a classic phenotype and contains a genetic mutation in either the mitochondrial DNA or the nuclear DNA. The mutations themselves are not sufficient to cause illness but are exacerbated by an environmental stimulus to promote disease development. The $1555 \mathrm{G}$ mitochondrial DNA mutation can cause decreased hearing or complete loss of hearing in many patients. When an individual patient is exposed to high levels of aminoglycoside, the risk of losing auditory function dramatically increases. This perfectly demonstrates how both nature, in the form of genetic mutation, and nurture, in the form of aminoglycoside exposure, are required for disease pathology.

A large percentage of mitochondrial patients are categorized as having possible mitochondrial disease. These patients present with a classic or less-than-classic mitochondrial phenotype but do not meet the criteria for any of the mitochondrial categories previously mentioned. Financial considerations, laboratory variants, and systematic collection error during examination are factors that contribute to the difficulty of diagnosing mitochondrial disorders.

In 1982, chromosomal karyotyping was the only viable option offered to clinicians to diagnose mitochondrial disease. With the advent of genetic technology, the ability of physicians to discern the genetic causes of disease has been widely enhanced. Genetic testing, mitochondrial DNA genome sequencing, and Next-Gen panels such as whole-exome and genome sequencing have dramatically improved diagnostic accuracy.

In many situations, muscle biopsies are thought to be too invasive but may be considered under certain circumstances when genetic testing is not feasible. In some cases, genetic testing yields inconclusive results or may not be available or be a feasible option because of insurance issues. Muscle biopsies can be used to confirm diagnosis when there is clinical evidence of a myopathy, neuropathy, or vasculopathy.

\section{Mitochondrial DNA Disorders}

Patrick Chinnery, PhD, Newcastle Research Group, Newcastle, United Kingdom 
Dr Chinnery opened by presenting a particularly interesting clinical case. A family brought in their 12-month-old son, who had unexplained encephalopathy. Muscle biopsy results were inconclusive. However, study of the respiratory chain complex showed a point mutation in complex $\mathrm{V}$, which is generally not tested in many laboratories around the globe. In complex $\mathrm{V}$, the clinical investigators discovered a point mutation at the 9176 position, which is important in the development of the ATPase-G protein.

Genetic testing was offered as a method to confirm diagnosis. This option was initially declined, because there was no family history of neurodegenerative diseases or mitochondrial DNA disorder. The family later reconsidered after the maternal aunt was affected with rapidly progressive blindness, a feature consistent with a mitochondrial DNA disorder. Dr Chinnery pointed out this demonstrates presentation of disease ranges widely, as the disorder can lie dormant until adulthood.

One copy of a genetic mutation, however, is not sufficient to produce disease in an individual patient. Dr Chinnery explained these kinds of disorders are characterized by heteroplasmy, in which there is often a mixture of mutated and wild-type genotypes within each individual. Mitochondrial disorders differ from Mendelian nuclear disorders in this aspect. This concept introduces the model of dosage of mutation and its effect on disease. Dosage of mutation can vary on the cellular, tissue, and organ level, illustrating differences in severity between 2 patients with similar genetic mutations. In reality, individual cells can tolerate high frequencies of genetic mutation; however, once the mutation level exceeds an established critical threshold, the biochemistry of the cell becomes compromised and could potentially result in the manifestation of mitochondrial disease.

Genetic variability is important to consider when establishing a diagnosis of mitochondrial disease. Dr Chinnery investigated the variability of mutation in a specific patient with a 3243 heteroplasmic mutation following a hysterectomy. After the surgical procedure, the investigative team obtained the ovaries from the uterus after micro-dissecting off the primary oocytes. With a sample number of 100 primary oocytes, the level and distribution of mutation within the oocytes was evaluated. From the study, scientists learned there is a range of potential heteroplasmic doses of mutation that can be passed on to future generations.

Embryonic development in mice was used as a model to observe the differentiation of mitochondrial DNA after germ cell fusion. As the embryo divides, the amount of mitochondrial DNA within each cell decreases, because there is no active replication of the mitochondrial DNA during cellular division. The decrease in mitochondrial DNA levels can be characterized as a genetic bottleneck. The sampling prenatal bottleneck effect acts to lower the chances of transmitting a high dosage of mutated mitochondrial DNA to future progeny. The information gathered from the oocyte mouse study can be translated for clinicians to illustrate the idea that these mitochondrial mutations that lead to disease in children are maternally inherited.

Prenatal diagnostic techniques are in the initial stages of development. Making an accurate diagnosis in utero is difficult, because heteroplasmy can vary over time and between 
individual cells. Performing a biopsy from amniocentesis or an embryonic preimplantation can provide untrustworthy results in terms of what will transpire during pregnancy. Therefore, providing guidance to families by describing a likely prognosis for their developing offspring is not always definitive. Currently, there are methods in development to prevent transmission of mutated mitochondrial DNA by transplanting nuclei during early embryonic development. Dr Douglas Turnbull from the University of Oregon published a study in which he was able to create a viable human blastocyst in vitro by swapping genetic information in the form of nuclei between donor and recipient embryos, thereby removing all mutated mitochondrial DNA. ${ }^{1}$

Numerous treatment options for patients are currently being studied. However, most of them are having little to no dramatic effect on the patient groups. In reviewing a database of more than a 1000 research articles published over the past 3 decades, only 35 published studies included a patient group larger than 5. Dr Chinnery's team reviewed the many publications that provided possible treatment options for patients and objectively scored the quality of the publications (Jadad scale, Oxford University). The Jadad scale, which ranges from 0 to 5, evaluates the quality of clinical trials on the basis of parameters such as randomization, blinding ability, and the effectiveness of how the methods were translated into the publication. From the study, Dr Chinnery noted a clear trend in which nonrandomized studies had lower and more significant $P$ values than randomized placebo-controlled trials. There is an increasing interest in developing future treatment methods for mitochondrial patients; however, Dr Chinnery discussed the need to change how scientists approach treatment development, to provide truly impactful studies.

In current studies, Dr Chinnery highlighted the fact that many scientists are trying to improve the quality of clinical trials. Interested groups are focusing on creating defined patient groups, building cohorts of patients that are larger in size, and randomizing and blinding studies to prevent clinicians from focusing specifically on positive results, which could in reality be false positives. Scientists also are working on developing clinically meaningful biomarkers to test mitochondrial function for future clinical studies. The North American Mitochondrial Disease Consortium is developing a patient database investigators will use to construct future studies. In the study of the database of clinical studies that have taken place from 1982 to 2012 as previously mentioned, the Jadad scale indicates that the quality of various clinical trials has been improving.

\section{POLG: Primary and Secondary Disorders}

Robert K. Naviaux, MD/PhD, University of California, San Diego, San Diego, California

In medicine, there has been a continuous debate between "nature versus nurture." Louis Pasteur studied microbial pathogens and labeled them as causative agents for disease. Claude Bernard, who is identified as the father of physiology, promoted the idea that it was the milieu, or the environment, that places human anatomy into a disequilibrium, which ultimately results in disease. The study of primary and secondary polymerase (DNAdirected) gamma disorders commonly referred to as "POLG disorders" indicates that in reality disease progression lies between the 2 scientific camps. 
Monogenic disorders represent 5\% of all known diseases. These classes of diseases are strongly dependent on a patient's genetic makeup as well as on the environment, which modifies disease severity. Such diseases include Alpers disease and ataxia neuropathy spectrum disorders. Counseling for monogenic disorders requires an extensive knowledge of the target genes and resulting metabolic disturbances that lead to physical clinical symptoms. Therefore, treatment of monogenic disorders specifically targets the disruption of normal metabolic pathways.

In contrast, ecogenetic disorders constitute approximately $95 \%$ of all known disease. Also known as complex disorders, the onset of ecogenetic disorders are initiated by a mix of genetic and environmental triggers. Examples of such disorders include autism spectrum disorders, valproate, stavudine, virus-sensitive ecogenetic variants, and various seizure disorders, as well as Tourette syndrome. One percent to $10 \%$ of these disorders fall under the subcategory of eco-dependent. In such cases, counseling requires intensive knowledge of life history and pathophysiology.

$P O L G$ is characterized as a mitochondrial DNA replisome located on the inner mitochondrial matrix. It is a heterodimer consisting of a catalytic $P O L G-1$ subunit and 2 regulatory accessory $P O L G-2$ subunits. In the human gene that produces the $P O L G$ replisome, there are more than 175 genetic loci vulnerable to mutation. More than 60 of these possible mutations can produce an Alpers disease phenotype. The vulnerability to produce mitochondrial disease is best illustrated by the fact that the allele frequency of pathogenic $P O L G$ mutations is approximately $2 \%$ of the human population as possible carriers.

Dr Naviaux described a clinical case of Alpers disease that he encountered during the initial interest in mitochondrial disorders. This particular patient of 1 year of age had a history of normal birth and early development prior to the visit. Five days after enduring an episode of infectious gastroenteritis, the patient began to present with decreased cognition that later was associated with seizures of mixed type with a prominent focal myoclonic component, and epilepsia partialis continua. Laboratory values revealed that the patient had multiple episodes of neurodegeneration associated with infection, hepatopathy, and elevated levels of cerebrospinal fluid protein. The disorder led to progressive cortical blindness, and the patient ultimately died at $3 \frac{1}{2}$ years of age.

In contrast, some $P O L G$ genotypes associated with Alpers-like disease may not present until later in life. In an example, a 73-year-old patient with a history of oculopharyngeal muscular dystrophy presented with Alpers-like symptoms. Histologic testing revealed he had ragged red fibers, ragged blue succinic dehydrogenase-positive fibers, and parking lot inclusions. It was later determined that the 73-year-old patient suffered from the same genetic mutation as the young child mentioned in the previous paragraph. The 2 scenarios, therefore, raise the question as to why disease onset differs between 2 individuals with the same genotype.

Ataxia neuropathy syndromes are mitochondrial disorders that affect teenagers and adult patients. The name changes according to the location where the POLG mutation has been established. Ataxia neuropathy syndromes include mitochondrial recessive ataxia syndrome 
or sensory ataxia and neuropathy, dysarthria, and ophthalmoplegia. Dr Naviaux focused on 2 common POLG genotypes: A467 T and W748 S. An interesting feature of these ataxia neuropathy syndrome genotypes is how the heterozygote version of the 2 alleles when they interact dramatically increases risk of mortality. In a study of 26 clinical cases, Dr Naviaux demonstrated how the A467T/W748 S heterozygote version decreases the survival rate of the various clinical cases in comparison with the 2 homozygotes, A467T/A467 T and W748S/W748 S. Dr Naviaux labeled this phenomenon as a heterozygote disadvantage.

Clinical overlap is a very common feature of many mitochondrial diseases. Dr Naviaux referenced 2 clinical studies by Hakonen, Soumalainen, and Tzoulis, Bindoff, respectively, that demonstrate this idea. In the 2 studies (with a combined $n=46$ ), 2 teams of scientists analyzed a variety of neurologic and metabolic phenotypes. In more than $90 \%$ of the cases, ataxia and dysarthria were present. Neuropathy, headache, hyporeflexia/areflexia, dementia, epilepsy, and nystagmus were also noted in a high percentage of patient cases. In a few cases, obesity, myoclonus, and psychiatric components were detected. Hepatic abnormalities were identified in more than $33 \%$ of patients.

Dr Naviaux presented the spectrum of $P O L G$ disease, describing 10 phenotypes with varying ranges of onset. High mortality rates were very specific to earlier-onset (birth to 20 years of age) diseases such as Leigh, Alpers, and myocerebrohepatopathy. The other 7 phenotypes were associated with a variable mortality rate. Adult presentations frequently feature multiple nonspecific prodromal symptoms. These can include dementia, headache, migraines, ataxia, and gastrointestinal dysmotility. Therefore, these symptoms frequently require additional symptoms in order for the clinician to begin to consider $P O L G$ as a possible etiology.

Reviewing more than 200 clinical cases, Drs Saneto and Naviaux analyzed the ages of onset of various $P O L G$ diseases. They discovered Alpers and MCHS are generally characterized by 3 peaks of onset during the early years (defined from birth to 20 years of age). The later Alpers peak is generally associated with homozygous forms of Alpers disease, specifically the A467 T homozygotes. They also investigated the various onsets of ataxia neuropathy syndrome and myoclonic epilepsy, epilepsy, and sensory ataxia starting from the beginning of adolescence into the 20 seconds. Later years (defined as 30-70 years of age) were characterized by onset of ataxia and multisystem cytopathies.

Dr Naviaux highlighted the fact that $P O L G$ diseases vary in expression over time. The temporary halt of disease progression and partial recovery are very common characteristics of both diseases. POLG disorders are often multiorgan diseases in which 3 or more organ systems are affected. The older age of onset is generally associated with a higher risk of compromised sensory neuropathy.

The last portion of Dr Naviaux's lecture was dedicated to covering the ecogenetic components associated with mitochondrial disease. Approximately 150000 years ago, the migration of mitochondrial DNA across the planet began. Each human population traveled to various locations throughout the world, where they established differing communities. Each of these communities exposed members of society to different climates, gastronomy, 
and microbial pathogens, so how mitochondrial DNA was affected by environmental agents differs among various ethnic groups. Only during the last 3 centuries has genetic information between various human populations begun to intermix. Ethnic mixtures such as Northern European and African groups migrating to North America propose a dilemma for scientists, as it is difficult to conduct ethnic boundary genetic studies in the United States without reference to ancestral origins.

Another important point Dr Naviaux discussed was that infection can act as a trigger to manifest a genetic disorder. In a study of 15 Alpers patients, $80 \%$ initially presented with neurologic or neurometabolic regression within 5 to 10 days of infection. Another study led by Dr Joseph Edmonds showed that of 40 clinical cases of severe childhood mitochondrial disease, $60 \%$ of the patients were in relative stasis or showing slow developmental progress, declining rapidly by degenerative events following infections.

Next-Gen metabolomics has given clinicians the ability to extract plasma or biological fluid or tissue, process it through a mass spectrophotometer, and measure a list of more than 800 metabolites in 63 metabolic pathways. Next-Gen metabolomics allows clinicians to convert metabolic data into perceptible clinical knowledge that can be used to develop novel and personalized methods of treatment. The logic behind how metabolomics function is through the realization that the human brain essentially controls metabolism. Every brain disorder produces a particular signature of irregularities that can be easily detected in blood or another biological fluid.

In summary, to properly diagnose primary $P O L G$ disease, the clinician can target the $P O L G$ gene or the POLG heterodimer itself with a compatible phenotype using a list of multiple technologies: mitochondrial DNA sequencing, long-range polymerase chain reaction (PCR), comparative genomics, and whole-exome sequencing. However, guiding the treatment of the patient requires physicians to perform a metabolomics test to determine the proper treatment. Clinicians must consider pathophysiology with modifiable risk factors and still use metabolomics to guide treatment of complex forms of the disorder, which constitute approximately $95 \%$ of cases.

\section{Question and Answer (Session I)}

Dr Maria: I have 2 questions, 1 for Dr Schon and 1 for Dr Cohen. Audience: We learned in training that in Kearns-Sayre, extraocular muscles were predominantly affected because of the heavy concentration of mitochondria in those muscles. Is that correct?

Dr Schon: Yes, heteroplasmy explains it. So those tissues that are affected have huge mitochondrial DNA mutation loads. The problem is we don't know why this distribution is so skewed. So to take Kearns-Sayre as a specific example, there is an incredible load of deleted mitochondrial genome in the choroid plexus. We have no idea why that is, but it does explain why one of the diagnostic criteria for KearnsSayre is elevated CSF protein, so the genetics are talking to you. What we don't understand is why selective mitochondrial concentrations are so different in different tissues. And that's true for some mutations and not others. So, for 
example, the mutation that Patrick talked about, 9176 and ATPase, that is pretty much spread uniformly throughout the body. It's very bizarre.

Dr Maria: You showed us a cocktail of therapies that we might all very much consider as standard therapy, but my question is, is there any real evidence upholding any of those individual therapies. Have you seen any kind of testing of those individual therapies that would give us confidence that they are in fact making a difference?

Dr Cohen: So Mark Tarnopolsky, who is going to be speaking later this afternoon, did a double-blind randomized placebo-controlled trial using CoQ10, creatine, and alpha lipoic acid, and it did show good $P$ values, clinical significance on biomarkers as well as physical outcome findings, so those 3 have been shown together. Now one could argue you ought to test each of them alone and separately. There's some good data that really becomes overwhelming that arginine and citrulline are helpful in preventing or decreasing the risk of stroke in patients with MELAS. Patrick has helped me out on a paper a few years where I had suggested using a folinic acid in patients with Alpers disease, and Patrick slapped my hand properly and said, "This is what we may do in clinic but you just can't put that in the paper yet because you don't have the clinical evidence to do so." So I've honed down my own personal recipe, and I do tell my patients that there is no overwhelming evidence that this works in the majority of patients, in fact, it doesn't work in the majority of patients, but I think Patrick ought to address that question as well.

Dr Chinnery: It's a difficult issue. Many of the treatments that Bruce is referring to are nontoxic and many of them are inexpensive, and so you can see how as a practicing clinician one would be tempted to use these medicines, and in fact, I do exactly the same thing. I suppose the concern is that if we keep practicing in that way, we won't move the field forward.

Audience: Do you have a sense as to whether viruses (versus bacteria) are more offensive, and whether it's the inflammatory response or whether it's actually the infectious agent that's causing the rapid decline.

Dr Naviaux: Viruses are more problematic than bacteria, and it's the host response that causes the problem more than the actual virulence factor associated with the pathogen. And so a lot of this is actually documented. If you take close clinical histories of declining patients with mitochondrial disease associated with infection, you're often surprised that really there are more neurodegenerative events that occur... . And part of that has to do with, it turns out that it's more bioenergetically demanding to heal than it is to acutely respond to environmental danger.

Dr Schon: Maybe I can add something to that, so it's become apparent in the last 4 to 6 years that there's a "signalosome" for immunity and infection that is located on the mitochondrial outer membrane in juxtaposition with the endoplasmic reticulum. For example, there's a gene called MAVS, mitochondrial associated viral signaling, 
a whole suite of proteins associated with that are upregulated only in the context of ER mitochondrial juxtaposition.

Audience: Valproic acid inhibits ureagenesis and it can cause hyperammonemia. But do you think that the toxicity is specific to $P O L G$ mutations or just any mitochondrial disorder could sensitize to valproate acid toxicity?

Dr Naviaux: So there are 2 answers to that. Valproic acid is a branch-chain fatty acid. It is a known inhibitor of mitochondrial function under the right conditions. It alters the biophysical properties of coupling in the organelle and isolated system, and it also depletes carnitine in children. Some adults will have hallucinations and new-onset seizures who were placed on it as a mood stabilizer.

Audience: Should clinical trials of new compounds be based on genotypes, phenotypes, or metabolomics?

Dr Chinnery: So I guess the simple answer is "all of the above." The problem is that they all introduce confounders in the clinical study for stratification, thus increasing the needed sample size. And I guess many of us have different views about this, and there's a really nice paper which will be relevant to you all in your practice, published by Shamima Rahman from Great Ormond Street, studying the natural history and mortality of patients with Leigh syndrome. It shows a striking difference in the prognosis for different biochemical and genetic forms of Leigh syndrome, which on presentation look very similar clinically. And it nicely illustrates the potential holes that we might fall into if we don't identify specific groups, which prior to her paper we didn't recognize, before we constructed these clinical trials. So I think my personal stance is that we need genetic homogeneity, which allows precise diagnosis. And whether one stratifies based on the severity of the biochemistry of that time point is an issue I don't think the field has come to grips with, and perhaps it should. And I suppose one way around this is to do some clever trial design with crossovers and placebos so that each patient acts as his or her own control.

Dr Cohen: I agree. I think we need to design trials whose entry point is a genetic diagnosis. Then once we get data that may be a specific phenotype within a genotype or vice versa, we can then home in on studying that better if we have a molecule to study.

Audience: A question for you Dr Schon and Dr Naviaux. Talking about why viruses are the causes as opposed to bacterial infection, we have something to treat the bacterial, whereas not much is available for viral. So the question is, is it because the antibiotic kills the bacteria so there's less of a burden to work on getting rid of that infection, whereas there's more of a burden with viral infections? Maybe seeing if viruses for which we have treatment are more often the cause, not often the cause, or usually not involved with this worsening might be helpful. What are your thoughts on that? 
Dr Naviaux: There are a limited number of ways that cells can respond with ultimate effect on interferon production. The anti-viral drugs used today are mitochondrial toxins like some of the antibiotics.

Dr Schon: I think the answer is yes, so there are deletions that are inherited. It's a little complicated because I didn't talk about the partner of the deletion, which is called the duplication. So imagine you had a 16000 -base wild-type genome, you took out 5000 bases. Now you have an 11000 -base genome. That genome can recombine at a low level to make a 26000 -base genome. And those actually can be inherited very nicely because they have no phenotype, because you have actually more than enough genes to go around on that circle.

\section{Molecular Mechanisms}

Moderator: William Copeland, PhD, NIEHS, Durham, North Carolina

\section{Defects of Mitochondrial DNA Replication}

William Copeland, PhD, National Institute of Environmental Health Sciences (NIEHS), Durham, North Carolina

Dr Copeland focused on the genetic aspect of mitochondrial research and how certain genetic mutations contribute to the pathology of disease.

Errors in mitochondrial DNA replication cause many of the clinical presentations of mitochondrial disorders. These mutations arise from various genetic sources, spontaneous errors in DNA replication, or damage by environmental agents. Some damage to the mitochondrial DNA is endogenous in origin, as the final by-products of oxidative phosphorylation in the electronic transport chain include the production of reactive oxidative species. The reactive oxidative species produced by the mitochondria can in turn damage the genome.

Maintenance of the human genome requires multiple classes of DNA polymerases to maintain proper function. Seventeen DNA polymerases that execute this important role have been identified. Of the 17 , only DNA polymerase gamma (DNA POLG) is responsible for mitochondrial replication and repair. DNA POLG functions in conjunction with a helicase TWINKLE, a topoisomerase, a single-stranded DNA binding protein, and various other genetic factors to reproduce the mitochondria's genetic information.

DNA POLG is a trimeric complex consisting of subunits from the POLG and POLG2 genes. $P O L G$ encodes for the catalytic subunit $\mathrm{p} 140$, which contains the primary DNA polymerase activity that processes the deoxyribonucleic acids replicate the mitochondrial DNA. The p140 subunit also contains a $3^{\prime}$ to $5^{\prime}$ exonuclease function, which aids in the proofreading ability of the protein in which it will correct any errors the polymerase may make during the replication process. p140 also contains a $5^{\prime}$-dRP lyase activity, which is essential in the basic incision repair process.

The gene POLG2 encodes the accessory subunits p55 dimers, which is critical in eliciting a high DNA binding affinity of the DNA POLG to the mitochondrial DNA. The dimeric 
proteins, p-55stimulate DNA synthesis and allow the complex to bind to the mitochondrial DNA during long periods of time. The p55 dimer is also labeled as the processivity factor, and it increases the processivity of the polymerase by several hundred-fold. The accessory subunit also is responsible for restoring salt tolerance by enhancing its ability to bind to the DNA template.

In a study conducted by Dr Copeland, the research copied a strand of mitochondrial DNA in vitro using purified DNA POLG and compared it with normal controls from patients in vivo. After matching the mutations to the control spectrum from the patients, the study discovered that of the genetic mutations identified, more than $90 \%$ of the genetic errors were mediated by DNA POLG. The results from the study suggest the repair processes present were absent or inefficient in the mitochondria. In general, DNA POLG acts as a very high-fidelity polymerase; in previous studies, it has been shown that it produces one genetic error for every million nucleotides incorporated during replication. However, it is believed that errors produced by DNA POLG are the sole source for producing spontaneous replication errors in the mitochondrial DNA.

A growing list of nuclear loci has been proved to contribute to the instability of the mitochondrial DNA. Errors in the replication of the many identified genes have been shown to lead to a multitude of differing diseases such as Alpers and ataxia; mitochondrial DNA depletion disorders produce progressive external ophthalmoplegia in children. When analyzing genes and their roles in mitochondrial disease, clinicians also have to look outside the mitochondria to the interacting proteins. For example, a defect in the gene that produces thymidine kinase II creates a blockade in the salvage pathway, which reduces the cell's pyridine storage, eventually leading to mitochondrial depletion-type syndromes such as mitochondrial neurogastrointestinal encephalopathy, also known as mitochondrial neurogastrointestinal encephalomyopathy (MNGIE).

POLG was not considered a disease locus until 2001, when Dr Gert van Goethem from Belgium was able to identify $4 P O L G$ mutations (3 recessive and 1 dominant) in his patients suffering from progressive external ophthalmoplegia. After this discovery, interest in the $P O L G$ gene dramatically increased in the scientific community. Currently, 238 genetic mutations within the $P O L G$ locus have been attributed to the development of mitochondrial disease. Of those mutations within the $P O L G$ gene, a number of mutated loci are concentrated around the active site of the enzyme believed to create a dominant negative enzyme, which is able to out-compete its wild-type counterpart. In general, early-onset diseases are associated with DNA depletion, whereas adult-onset diseases are usually activated by DNA deletions.

Presently, mitochondrial specialists are turning to yeast genetics to study more about mitochondrial DNA replication and metabolism. To date, approximately 50 mutations that are conserved between humans and yeast have been identified. Petite yeasts are unable to incubate on media with nonfermentable sources of carbon, because of a defect in the respiratory chain. Only in the presence of a fermentable carbon source, such as glucose, can the yeast establish proper growth of an anaerobic colony. Using these petite assays, yeast 
genetics is able to easily screen for mitochondrial dysfunctions, such as mitochondrial DNA depletion, point mutation, and deletions.

There are 2 allelic forms to consider while performing genetic assays. An assay that contains the heteroallelic form includes the wild-type and mutant copy of the chromosome on a plasmid that elicits a heterozygous situation. In contrast, the monoallelic form of the wildtype copy is knocked out, so the only viable copy left is the mutated allele. This emulates a homozygous situation within the cell, so the role of the differing mutation can be analyzed and assessed. Of the disease mutations, most genetic errors result in the loss of mitochondrial DNA and, by association, a loss of respiratory activity. This is seen in the increase in petite colony formation initiated by the knockout of the wild-type allele.

The $3^{\prime}$ to $5^{\prime}$ exonuclease performs a proofreading function when DNA PolG creates an error during mitochondrial DNA replication. A previous study conducted by Dr Larson in Sweden created an exonuclease-deficient DNA PolG mouse by knocking out the wild-type alleles and then reintroducing either a heterozygous or homozygous $5^{\prime}$ to $3^{\prime}$ exonuclease-deficient mouse. Dr Larson discovered that the heterozygous $5^{\prime}$ to $3^{\prime}$ exonuclease-deficient mice were asymptomatic through life; however, homozygous $5^{\prime}$ to $3^{\prime}$ exonuclease-deficient mice presented with premature aging and died at 1 year of life.

Another group of scientists used the same protocol, testing the differing mutation levels between the 2 murine models. They discovered that the homozygous POLG exonuclease -/exhibited a 2000-fold increase in point mutation as opposed to only a 500-fold increase the heterozygous POLG exonuclease $+/-$. The heterozygous POLG exonuclease $+/-$ deficient mice did not display a defective phenotype, proving that mitochondrial DNA is exceptionally tolerant to random point mutation up to an established threshold.

DNA POLG under normal circumstances creates low-frequency point mutation errors. However, an activated exonuclease will correct the base mismatch or other replication errors. When the exonuclease activity is absent or has been knocked out, the DNA POLG will incorporate mismatched bases uncontrollably. If the mutation is located in an area of a direct repeat, this can result in a slippage event by the polymerase on the mitochondrial DNA, which results in the formation of a base deletion. An example of this occurs in Kearns-Sayre and PEO patients in which there is a 4977-base pair deletion that is commonly seen in these patient populations.

Another crucial component of DNA POLG is p55, also known as the accessory subunit of the polymerase. The p55 subunit acts as a dimeric protein that contributes to the overall processivity of the DNA polymerase. The accessory subunit is not conserved throughout the eukaryotic phylogeny, as it is absent in yeast, single eukaryotes, and various species of nematodes. In invertebrates such as insects, it exists in a monomeric form, whereas in high vertebrates, specifically in mammalian species, it is present as a dimer.

Using a murine model, Dr Copeland's team crossed 2 heterozygous knockouts POLG2 $2^{-/+}$ mice to form a homozygous knockout POLG2 ${ }^{-/}$. Unfortunately, upon birth, there were zero survivors, suggesting that the homozygous knockout results in embryonic lethality.

Retracing the gestational period, it was determined that development of the POLG2 ${ }^{-/-}$ 
embryo had been arrested at day 8.5. Looking at histologic evidence, in the POLG2 ${ }^{-/-}$ homozygous mouse there was an abrupt loss of COX activity, which is critical to cellular development, whereas the POLG2 $2^{-/+}$heterozygous displayed normal wild-type development. In addition, there were noticeable structural defects detected in the POLG2 $2^{-/-}$ mouse embryo. Cardiac tissue taken from the homozygous knockout displayed swollen mitochondria, a loss of cristae (demonstrating a loss of the inner mitochondrial membrane), and an increased accumulation of lipids, because of a loss of fatty acid oxidation activity within the mitochondria.

The general findings from Dr Copeland's murine study underline the importance of the p55 accessory subunit to the viability of life within larger complex eukaryotes. From the study, the heterozygous POLG2 ${ }^{-/+}$knockout was viable from birth to up to 2 years of life. Realtime PCR quantification of mouse mitochondrial DNA demonstrated a decreased amount of mitochondrial DNA in the POLG2 ${ }^{-/+}$heterozygotes and a complete loss of mitochondrial DNA in the POLG2 ${ }^{-/}$mice. Ongoing research is testing the level of vulnerability of these heterozygous mice to environmental stressors such as reactive oxidative species.

There is an emerging list of genetic mutations occurring in conserved areas of the human genome. One of these is the G451E mutation, which was originally identified by Dr Patrick Chinnery. From a replication assay, the products were separated on an agarose gel. The gel displayed by Dr Copeland suggests that the association of the p55 accessory dimer to the catalytic p140 subunit is essential to the overall processivity of the enzyme. An experimental issue is that the tests run in vitro are homodimers, whereas in vivo the 2 accessory subunits are heterodimers. Generally, all heterodimeric p55 proteins are able to adequately bind to the p14 catalytic subunits. To correct for this difference, Dr Copeland developed an assay that is able to use tagged subunits to isolate heterozygous populations of mutant and wild-type $P O L G 2$ heterodimers.

After replicating all the assays with the renewed tagged subunits, Dr Copeland demonstrated that when a mutant allele is crossed with a wild-type allele, normal replication results, signifying that the mutation is recessive and can be rectified when crossing with a wild-type allele. The G451E mutation causes the p55 subunit to be unable to bind properly to the p14 catalytic subunit. This situation creates a dominant negative enzyme that outcompetes the wild-type polymerase; therefore, this specific mutation, unlike the previously studied genetic errors, is inherited in an autosomal dominant fashion.

Presently many researchers are using cell-based models to overexpress mutant variants in tissue culture cells. In a study designed to view the localization of the p55 variants, Dr Copeland used 4',6-diamidino-2-phenylindole staining and counterstained the p55 wild-type with downstream regulatory element antagonist modulator fluorescent protein. Dr Copeland discovered that removing the mitochondrial leader sequence from the targeted gene results in a diffused staining throughout the cytoplasm. Removal of the leader sequence emulates a delocalization situation of the 555 protein within the cell, denoting the leader sequence's importance in the localization process. 
Dr Copeland ended his discussion by underlying the importance of testing mutated genes with multiple assays to properly understand the pathology of the growing list of genetic mutations discovered within the mitochondrial DNA.

\section{Mitochondrial Dynamics and Parkinson}

Richard Youle, PhD, National Institute of Neurological Disorders and Stroke (NINDS), Bethesda, Maryland

Continuing with the theme of molecular mechanisms of disease, Dr Richard Youle presented his research at the NDC Symposium on the genetic bases of Parkinson disease. Dr Youle discussed the scientific community's growing interest in the role of the mitochondria and the mitochondrial quality control pathways in the neurodegeneration and progression of disease within Parkinson patients.

Most late-onset cases of Parkinson disease have little symmetry of distribution between siblings, indicating there is little genetic basis for disease development. For early-onset cases, there are a number of monogenic causes of disease progression. Dr Youle focuses on 2 prominent genes, parkin and pinkl. Mutations in these 2 nuclear genes follow an autosomal recessive pattern of inheritance, indicating they are most likely a loss-of-function mutation. By determining the gene products of parkin and pinkl, scientists could gain insight into the cellular mechanism and cause of disease, which could inspire the development of novel therapeutics.

The 2 genes were initially cloned and their sequence was analyzed to ascertain the function and role of the gene products. Dr Youle discovered that parkin generates an E3 ubiquitin ligase, which previous studies have shown to be important in protein degradation. The pink1 gene translated a PINK1 protein that contains a kinase domain, in addition to a sequence that is important for mitochondrial import and localization. PINK1 is a mitochondrial kinase that targets damaged mitochondria when there is a loss of membrane potential or an increase in reactive oxidative species damage. Once PINK1 accumulates within the mitochondria, it will actively recruit the E3 ubiquitin ligase to mark the mitochondria for mitophagy by an autophagasome. The mitochondria will accumulate damage from an endogenous source from DNA mutations or from toxic environmental agents. If these toxins or mutations accumulate to a certain threshold, it will cause depolarization of the outer mitochondrial matrix, which will in turn trigger the PINK1 kinase and the subsequent mitochondrial quality control pathway. This will ultimately result in the degradation of tagged mitochondria by autophagosomes that engulf the organelles, fuse with surrounding lysosomes, and digest the impaired organelle.

The mechanism previously described, if established to be true in vivo, presents an interesting plan of attack for many pharmaceutical companies looking to develop treatments for Parkinson patients. It is thought if this particular mitochondrial quality control pathway is augmented, pharmaceutical companies can create strategies to purify and eliminate the accumulation of deleterious mutant mitochondria that is theorized to play a crucial role in the pathology of Parkinson disease. 
The PINK1 kinase is translated in the cytosol and then introduced into the mitochondria via importation through the TOM and TIN pathways. In the inner membrane space, an enzyme labeled PARL constitutively degrades PINK1 kinase in healthy mitochondria. If there is a deleterious event that impairs the oxidative phosphorylation pathway, the importation of PINK1 kinase will be inhibited, which will lead to a loss of membrane potential. Because the PINK1 kinase is not imported and therefore does not associate with the PARL protein, the kinase will accumulate in high amounts on the outer membrane of the mitochondria. Uncontrolled phosphorylation on the outer membrane of the mitochondria results in the recruitment of the parkin protein E3-ubiquitin ligase to the surface of the impaired mitochondrion and establishes an ubiquitination event for elimination mediated by a mitophagosome.

In a subsequent study, Dr Youle's team fused the E3-ubiquitin ligase to green fluorescent protein, which will show up against a red mito tracker dye under the view of an electronic microscope. By biochemical and genetic methods, Dr Youle was able to uncouple the proton gradient important in the final stages of oxidative phosphorylation, thereby triggering a depolarizing event on the outer membrane of the mitochondria. Depolarization of the proton gradient initiated the accumulation of green fluorescent protein on the surface of the mitochondrion.

Dr Youle, with the collaboration of Dr Giovanni Manfredi, used the blood plasma of a patient with a cytochrome $\mathrm{C}$ oxidase mutation and created genetic cybrids. Dr Manfredi extracted the plasma and fused the cells with a normal human cell line and expressed green fluorescent protein-tagged parkin protein. Without using an uncoupler protein, the parkin protein localized specifically to the damaged mitochondria. Therefore, pinkl is able to sense and filter out the damaged mitochondria and will consequently recruit parkin protein to specifically localize to the impaired mitochondria.

By expressing a genetic vector in the cell line that contains $80 \%$ mutated rate for more than 45 days, there is no change in the degree of heteroplasmin within the cell line. However, by increasing the level of E-3 ubiquitin ligase, the mutant cell line diminishes over an additional treatment period of 45 days. Extending the treatment to 60 days almost completely eliminates the level of mutated mitochondria, indicating that the gene parkin protein mediates the quality control pathway within the mitochondria.

RNA interference (RNAi) screening has played a crucial role in finding new genes that interact with various pathways. Dr Youle and his team used a high-content image analysis, and treated cells in 384 wells with an uncoupler to trigger E-3 ubiquitin ligase to the mitochondria. Midway through the procedure, Dr Youle's team introduced a series of 83 000 RNAis into the individual wells targeting a variety of genes to block. They screened thousands of RNAis to discover new genes. Some of the targeted genes, if you knock them out, will inhibit parkin protein translocation, such as the gene products from TOM7. During the study, Dr Youle and his team identified 2 RNAi libraries: the Dharmacon library and the Andean library, which contains 3 unique RNAis per gene. 
A problem when dealing with RNAi screening technology is there are unfortunate off-target effects of using RNAis. Therefore, how do investigators know what they are testing is not an off-target side effect? New advances have appeared in biological technology, genome editing using transcription activator-like effector nucleases (TALENs), and clustered, regularly interspaced, short palindromic repeats (CRISPRs), which assess targeted genes without confounding off-target influences. TALEN and CRISPR technology also expedites the process of screening the genome in an RNAi-independent method. Using this new technology, the team is able to completely knock out the TOM7 gene, which results in a thorough prevention of E-3 ubiquitin ligase translocation. This blockage of parkin increases the level of mutated or impaired mitochondria within the cell line. By reintroducing the TOM7 back in, the wild-type phenotype can be rescued.

The previously mentioned studies have focused mainly on in vitro models, so Dr Youle segued his discussion to testing in vivo the role of parkin and pinkl using a mouse model. In this particular study, Dr Youle crossed a POLG gene mutated mouse $\left(\mathrm{POLG}^{-/}\right)$with a parkin knockout $(p \mathrm{KO})$ mouse. They then studied the neurologic histology of progeny from such a cross. Dr Youle quantified the decrease in substantia nigra neurons in wild-type, $\mathrm{POLG}^{-/-}$mutator, the $p \mathrm{KO}$, and the $\mathrm{POLG}^{--} p \mathrm{KO}$ cross progeny. In the analysis, there was no loss in the wild-type, $\mathrm{POLG}^{-/-}$, or the $p \mathrm{KO}$ mutated mice. However, the study detected a $45 \%$ decrease in the number of substantia nigra neurons in the $\mathrm{POLG}^{-/} \times p \mathrm{KO}$ cross. This finding indicates that the endogenous parkin protein is alleviating the loss of substantia nigra neurons in the mutated forms.

Dr Youle also focused on movement disorder in the mice by performing a poll test, which assesses each mouse's ability to travel down a pole. Of the other tested groups (10 seconds latency) in the study, the $\mathrm{POLG}^{-/-} \times p \mathrm{KO}$ cross was the only group to have a significant increase (50 seconds latency) in the latency before they were able to climb down the poll. This is consistent with the loss of dopaminergic neurons that this test group suffers from. To test whether the latency is accurately attributable to the loss of substantia nigra neurons, Dr Youle performed another test trial by treating the $\mathrm{POLG}^{-/} \times p \mathrm{KO}$ cross mice with ${ }_{\mathrm{L}}$-dopa $(100 \mathrm{mg} / \mathrm{kg})$. Treatment with ${ }_{\mathrm{L}}$-dopa completely mitigated the increased latency this specific cross was suffering from. The crossed mutated mice that were not treated with $\mathrm{L}_{\mathrm{-}}$-dopa were assessed a month later. The latency had increased on average to 150 seconds. However, this was also reversed via treatment with $\mathrm{L}_{\mathrm{L}} \mathrm{dopa}$, indicating that the motor-deficient phenotype is caused by a loss of substantia nigra dopaminergic neurons, which proves that endogenous E-3 ubiquitin ligase relieves the damage from mitochondrial DNA mutation accumulation within the mitochondrial organelle.

By viewing the pathology of diseases such as Parkinson, it is evident that damage to the mitochondria is not just mediated by outside damage or genetic mutation, but also by impairing essential conserved pathways such as the quality control pathway, which effectively eliminates and prevents the accumulation of compromised mitochondria. Future research in treatment is looking to increase the expression of parkin or the activity of E-3 ubiquitin ligase to alleviate the effects of disease in patients. 


\section{Systems Biology Approach to Mitochondrial Disease}

Vamsi Mootha, MD, Harvard University School of Medicine, Boston, Massachusetts

Dr Mootha introduced the computational biology approach to mitochondrial research, specifically the mito exome sequencing method that has helped promote the advancement of research into mitochondrial medicine. A large portion of the findings that he discussed during his lecture was done in close collaboration with Dr David Thorburn from Australia and a fellow colleague at Harvard University, Dr Sarah Calvo.

The heterogenetic phenotype that characterizes mitochondrial disorders makes these illnesses difficult to diagnose. Most mitochondrial cases are multisystemic diseases that affect multiple organ systems. Leber hereditary optic neuropathy, for example, presents with deep gray matter lesions, myopathies, eye disease, and anemia. Other cases could include peripheral neuropathy, liver failure, and progressive external ophthalmoplegia (PEO).

A major landmark event for systems biology occurred in 1981, when the sequencing of the mitochondrial genome was completed. These sequences provided a molecular basis to expose the genetic causes of many but not all maternally inherited mitochondrial disorders. The mitochondrial organelle is constructed using both the mitochondrial and nuclear genomes. The mitochondrial DNA encode the 13 essential proteins required for proper oxidative phosphorylation. Another 77 subunits, however, originate from the nuclear genome. Therefore, understanding this disorder requires a solid understanding of both nuclear and mitochondrial genomes. To translate the 13 proteins from the mitochondrial DNA, there are essential cofactors that are derived from the nuclear genetic material. DNA PolG is a polymerase that is encoded by the nuclear genome but plays a crucial role in the replication of mitochondrial DNA. This interaction is a testament of the ongoing symbiotic relationship that exists between the eukaryotic cell and its "prokaryotic" organelle.

Dr Mootha, using mass spectrometry proteomics, constructed an inventory of 1100 proteins encoded by the nuclear genome that, in collaboration with the mitochondria's 13 essential proteins, comprise the mitochondrial proteome called MitoCarta. This inventory was constructed using proteomic and computational methods, a literature review of previous studies, and green fluorescent protein microscopy. In the laboratory, Dr Mootha's team extracted biologic tissue from 14 mice, isolating the 1100 proteins with subtractive mass spectrometry and filtering the list with Bayesian integration.

The MitoCarta has proved to be a useful tool for many clinicians. Dr Mootha presented a patient case in which a young female patient with Leigh syndrome presented with lactic acidosis, and from a muscle biopsy it was ascertained that she suffered from an isolated complex I deficiency. Her parents were first cousins and she had an affected older sibling. In such a case, the clinician is able to perform homozygosity mapping, pedigree mapping, and linkage analysis to determine the region of the human genome that harbors the mutated gene. From this information, Dr Mootha crossed it with the MitoCarta inventory to see which of the 1100 proteins are located within this genetic interval, and the specified disease gene was elucidated for clinical diagnosis. 
Still, even with the advancement of the MitoCarta, clinicians question which of the many genes should be tested in a given single patient. The process is still inefficient, and much work is being focused on improving diagnosis. Fortunately, since 1988 the process has been steadily improving.

Genetic sequencing has become incredibly more efficient and inexpensive since the passage of Moore's Law, which states that the cost of computing sequences should decrease 2-fold every 18 months. During the past 11 years, the cost of sequencing has dropped a millionfold, driving new opportunities in the area of clinical research.

Another component of the MitoCarta project included the introduction of MitoExome sequencing, which uses novel technologies such as next-generation sequencing to decode the mitochondrial genome, which will help identify the specific genetic abnormalities that are causing disease in mitochondrial disease patients. Presently, Dr Mootha and Dr Calvo have conducted MitoExome sequencing on 3 cohorts of patients from varying levels on the disease spectrum. During the sequencing of the cohorts, Dr Mootha was able to identify 8 new disease-causing genes.

The focus of the patient study assessed the efficacy of the next-generation sequencing approach to provide a genetic method to diagnosis. Of the 3 cohorts studied, the third consisted of a broader phenotypic spectrum; therefore, clinical diagnosis was more challenging. Many of their clinical features were suggestive for mitochondrial disease, but the muscle biopsies did not provide strong evidence of a mitochondrial disorder. In addition to the wider phenotypic range in this particular cohort, the age distribution of the patients also spanned a larger range. The third cohort contained 102 patients, including 18 controls of patients who had already received a definitive molecular diagnosis of disease. Out of the 102 patients studied, 84 were labeled as having an unknown diagnosis.

Of these 84, Dr Mootha presented one clinical case in which a 3-month-old girl was affected with severe pulmonary hypertension, microcephaly, hypotonia, and basal ganglia calcifications. The patient demonstrated elevated alanine and lactate levels, and had undergone a muscle biopsy that indicated a combined respiratory chain deficiency. Initially, Dr Mootha assessed the patient with a variety of mitochondrial DNA depletion-deletion panels, which displayed inconclusive results. Next, MitoExome sequencing was applied and indicated various recessive mutations in ATP5Al. This mutation was determined to be a point mutation that created a nonconcerted change from tyrosine to cysteine, which affects the ability of complex V (ATPase) to function properly in the mitochondrial respiratory chain.

A second case that Dr Mootha presented included a 4-year-old girl who suffered from a neurologic condition. The symptomatic presentation included myoclonic jerks, dystonia, and frequent episodes of chronic fatigue. It was determined through biochemical testing that the patient only expressed $14 \%$ of normalized complex I and III activity; therefore, $86 \%$ of the nascent function was absent in the patient. From the MitoExome sequencing, it was determined that there was a homozygous splice mutation in dihydropyrimidine dehydrogenase that is involved in nucleotide metabolism within the cytosol. Usually 
dihydropyrimidine dehydrogenase deficiency is not classically classified as a mitochondrial disease; however, the patient exhibited some biochemical evidence that was suggestive of mitochondrial disease pathology. This particular case was ultimately labeled as a secondary mitochondrial dysfunction.

In the third cohort, MitoExome sequencing was able to establish 6 new diagnoses out of the 84 unknown cases, relating to the fact some mitochondrial diseases are often incredibly difficult to diagnose, even with access to advanced technological methods.

Over the last 6 years, Dr Mootha analyzed the exome sequences of more than 200 patients. Of those assessed, $20 \%$ of the cases were associated with a confident diagnosis of mitochondrial disease without the need to perform a muscle biopsy. Another $20 \%$ was determined to have a recessive cause of disease. In conclusion, $60 \%$ of clinical cases remain unsolved. The reason could be attributed to the ability of MitoExome sequencing to detect only monogenic causes of disease. The etiology of many mitochondrial illnesses is initiated through 2 or more genes working in collaboration to establish disease pathology. Some of the causes may not be based in genetics. With an increasing number of studies devoted to mitochondrial disease development, emphasis is starting to be placed on environmental agents that trigger disease onset.

A 35-year-old man displayed symptoms concurrent with a progressive neurologic disorder. Presentation of disease began with severe cerebellar ataxia by age 29 and complete loss of hearing by age 34 . A muscle biopsy indicated a combined respiratory chain deficiency. After an initial completion of MitoExome sequencing, there were no single nucleotide variants that could be considered causal candidates of disease. After reviewing the analysis, $\mathrm{Dr}$ Mootha discovered a copy number variant. A deletion in a paroxysmal protein HSD17B4 was attributed to be the causative agent of disease. The patient was ultimately diagnosed with "probable" mitochondrial disease.

MitoExome sequencing has changed the way many clinicians can now diagnose disease, without invasive procedures such as muscle biopsies. Dr Mootha ended his discussion by reinforcing this message, saying there is much improvement in diagnostic methods in the field of computational biology. Dr Mootha stated that in reality most methods available have only 50\% sensitivity, which signifies that half of the positive screens that are executed could be false positive results, underlying the need for prospective studies that will improve the utility of computational technology in the field of mitochondrial medicine.

\section{Question and Answer (Session II)}

Audience: Could regional mosaicism account for some of the difficulty in establishing a genetic diagnosis, and that you really need to access samples from the most impaired tissue to confirm the genetic ideology? That's the first question. And my second question is, why cannot the cell that has, for example, three quarters of abnormal mitochondria not rely on reproduction of the normal mitochondria to overcome the metabolic defect of the cell? And is there any evidence that there's some sort of toxic effect from this genetic condition that 
impairs replication of normal mitochondria and may even account for some of the degenerative flavor of the disease?

Dr Mootha: I'm happy to respond to the first question. I think mosaicism could definitely contribute to difficulty in establishing a diagnosis. And that's what I refer to when I talked about somatic genetic causes of the diseases. One could imagine with aging mutations either in the mitochondrial DNA or in the nuclear DNA that might be compounded with an inherited mutation. So I think the answer is definitely yes, but it's going to require that we start interrogating the target tissues as well, but the mitochondrial DNA as well as the nuclear genome.

Dr Copeland: In terms of the second question, we definitely do see compensatory mechanisms when you have defective replication. The problem with compensatory mechanisms as Eric Schon pointed out, you can't have selective, upregulation of just the wild-type copy or good copies of mitochondria of DNA relative to the mutant ones. There's just no mechanism to differentiate that. So compensatory mechanisms usually upregulate everything. Now if you can treat it with a drug or something like Vazotan and you upregulate everything to a threshold where you now have enough wild-type copies at some absolute level, then you're fine. It doesn't matter how many bad copies there are, but there are.

Part of your question was is there toxic side effects to mutant, I mean other than loss ...

Audience: Yes.

Dr Copeland: Loss of ATP, I don't know if you know of any ...

Dr Youle: I can't think of any but it's actually an interesting proposal worth thinking about.

Dr Mootha: Yeah, like a by-product or something.

Dr Youle: Right, like cardiolipin or some metabolic product. It could be. That's good.

Dr Mootha: Definitely. Possibly.

Audience: I've a PINK Parkin question. What happens if you use just the right amount of oligomycin to hyperpolarize mitochondria?

Dr Youle: You can inhibit the pathway, and in fact normally if you have one impaired mitochondria, it can actually use the ATP ACE cleaving ATP to generate enough membrane potential to prevent PINK1 accumulation and prevent mitophagy. So there are likely other steps. We have a paper, just came out, showing misfolded proteins in the mitochondria can induce Parkin accumulation and actually purify the misfolded proteins without loss of membrane potential. I think there's other steps, maybe more regulatory, in controlling the TOM/TIM pathway for import of PINK1 above and beyond membrane potential, but I didn't have time to go into all that. 
Audience: I've got a question for Dr Mootha. Can you tell us what's happening on a national or international consortium level to store and analyze all the normal and abnormal whole-exome sequences?

Dr Mootha: That's a great question. So I know that these 2 efforts are ongoing that I think are really exciting and are going to help to move the field forward, both from a research standpoint as well as from a clinical standpoint. And in genetics right now and genomics, the real key is data aggregation. Our ability to interpret a single genome is incredibly difficult, but imagine a world in which you have a look-up table of thousands of genomes from patients as well as millions of genomes from "healthy controls"; it would basically be an afternoon exercise to then try to spotlight which are causal for disease versus innocent bystanders. So across various different disease communities, including our own, the mitochondrial disease community, there are now data aggregation efforts. And I know that Marni Falk is leading what I think is a very important effort to try to aggregate "morbid" exomes from patients with mitochondrial disease.

And more broadly, there is something called the Global Alliance. This was just announced a few months ago. This is a very large initiative that has buy-in from the Sanger Center, various cancer centers around the United States, around the world. Several heads of nations, heads of state have also signed on. It's a multinational, multiinstitutional agreement to try to create a cloud space in which exomes and genomes could be uploaded in a manner that is very protective to patients while also promoting data exchange, clinical interpretation, and research. And so these are 2 activities, The Global Alliance as well as the North American Mitochondrial Disease Consortium (NAMDC), in the broader community as well as in the mito community that I think are absolutely essential for moving the field forward.

\section{Translational Science and Clinical Frontiers}

Moderator: Greg Enns, PhD, Stanford University School of Medicine, Palo Alto, California

\section{Gene Therapy and Enzyme Replacement}

Michio Hirano, MD, Columbia University Medical Center, New York, New York

Dr Michio Hirano covered current research on gene therapy and described enzyme replacement of a nuclear DNA defect of a particular patient suffering from a mitochondrial anomaly.

As mentioned earlier during the NDC Conference, most genetic mutations within the mitochondrial genome are heteroplastic in nature, meaning there is usually a mixture of wild-type and mutant mitochondrial DNA that characterizes each patient's genetic material. At times, this complicates clinicians' ability to diagnose disease. However, Dr Hirano viewed this particular pathology as a potential avenue to construct a novel treatment. Dr Hirano proposed developing a technique to shift the heteroplasmy of affected cellular tissue during maternal transmission of genetic material. 
Patients suffering from an 8993 point mutation in the complex V-ATPase gene result in 2 clinical phenotypes. Depending on the degree of mutated mitochondrial DNA within the patients' cells, the mutation could lead to severe basal ganglion lesions commonly found in Leigh syndrome or milder to asymptomatic forms of disease. In general, more severe forms of disease contain a higher level of mutation ( $90 \%$ or more) and milder forms have an intermediate level (between 70\% and 90\%), whereas asymptomatic carriers of disease have the lowest concentration of mutated genetic material (generally below 70\%). By shifting the level of mutated mitochondrial DNA under the established disease threshold, one could theoretically establish an asymptomatic carrier of disease.

The maternally inherited Leigh syndrome (MILS) mutation (T8993G) incorrectly incorporates a guanosine nucleotide in the place of a thymine, thereby creating a unique Smal restriction enzyme site. By incorporating the MILS mutation in other disease loci, it is now possible to import a restriction enzyme to eliminate mutant mitochondrial DNAs selectively.

Dr Hirano referenced a study conducted by Dr Michal Minczuk, who was able to develop single-chain zinc finger nucleases for selective degradation of mutated mitochondrial DNA. In the referenced study, Dr Minczuk was able to use the zinc nuclease to bind selectively to mutated ATPase6 gene and effectively. In cultured cells, Dr Minczuk was able to shift the mutated level from $80 \%$ to $70 \%$. $^{2}$ Another gene therapy option has recently refined the use of zinc finger nucleases by using transcription activator-like effector nucleases, which are better able to localize specifically to the mitochondria. In addition, transcription activatorlike effector nucleases are able to more effectively shift the level of heteroplasmy to under $40 \%$ mutated level, which under most cases results as asymptomatic. This development of transcription activator-like effector nucleases raises the possibility for these particular mitochondrial-specific nucleases to become a new affective therapeutic option for reducing severity of mitochondrial disease. ${ }^{3}$

Another developing technique that was discussed was pronuclear transfer in human embryo to prevent transmission of mitochondrial DNA variants. This therapeutic strategy included extracting a donor nucleus from an embryo and implanting the nucleus in an affected zygote. By introducing a wild-type nucleus into a recipient zygote, the technique purifies the nuclear DNA of any mutated mitochondrial DNA that was present. The levels of mutant mitochondrial DNA of the reconstituted zygote should be void or at very low levels. This experiment was also translated from zygotes onto the monocyte level, which later resulted in embryos that were able to differentiate and propagate cells that retained wild-type mitochondrial function.

Mitochondrial neurogastrointestinal encephalomyopathy, is a mitochondrial disease that is characterized by both depletion and deletion of mitochondrial DNA. Patients suffering from this class of mitochondrial disease contain a loss of function mutation in the TYMP gene that encodes the enzyme thymidine phosphorylase, which is important in the degradation of the nucleocyte thymidine to its constituent base (thymine) and sugar ribose. With improper enzyme activity, cells accumulate toxic metabolites in the cytosol, which enter the mitochondria, where they will be phosphorylated, creating thymidine triphosphate (TTP) 
and uridine monophosphate (UMP) precursors. The nucleotide precursors are later incorporated into the mitochondrial genome. The accumulation of thymidine and other toxic base metabolites creates an imbalance of nucleotide triphosphates and leads to secondary mitochondrial DNA mutations that will accumulate over the patient's life span.

In treating mitochondrial neurogastrointestinal encephalomyopathy, many therapeutic options have been considered and assessed. Dialysis to remove noxious metabolites and prescribing drugs to increase renal excretion of excess thymidine and deoxyuridine were assessed to test the efficacy of treatment. Unfortunately, both methods resulted in providing no relief for the patient or affective decrease in thymidine or deoxyuridine levels.

Enzyme replacement therapy is another treatment option currently being considered. This treatment strategy extracts red blood cells from patients, and places them in a hypotonic solution. The red blood cells in the hypotonic solution will swell, increasing the size of their pores on the apical surface, allowing thymidine phosphorylase in the solution to enter the red blood cells. After the red blood cells encapsulate, the thymidine phosphorylase will be infused back into the patient. Toxic thymidine levels were subsequently assessed and decreased after treatment.

Dr Hirano and his team also are evaluating a treatment strategy that infuses platelets rich in thymidine phosphorylase back into affected patients. The infused platelets were introduced into a patient suffering from mitochondrial neurogastrointestinal encephalomyopathy, loss of enzyme activity was restored, and levels of toxic metabolites were reduced over a period of 7 days.

Dr Hirano presented a case of a 30-year-old female patient with mitochondrial neurogastrointestinal encephalomyopathy who had undergone donor transplant therapy. Initially she presented with prominent gastrointestinal abnormalities, abdominal pain, recurrent diarrhea, and myopathy with notable muscle weakness. After the transplant, disease symptoms were relieved, muscle strength regained, and thymidine phosphorylase restored to normal function. This same therapy was attempted in 26 patients. Only 9 of the therapies have proved successful and 7 patients died after treatment.

Therapy for mitochondrial disorders continues to advance, and new treatment strategies are becoming available. A major new development is the establishment of the North American Disease Consortium (NAMDC), which is sponsored by the National Institutes of Health. The North American Disease Consortium currently consists of 18 sites across the North American continent and is planning to design a future registry of patients who have mitochondrial disease. The registry will include patient information along with biological samples that can be used in future treatment studies.

\section{Exercise Therapies in Attenuating Mitochondrial Disease}

\section{Mark Tarnopolsky, MD/PhD, McMaster University, Ontario, Canada}

Dr Tarnopolsky presented an extensive overview of the potential benefits of exercise for patients who have mitochondrial disease. The muscle tissue relies on the inner mitochondrial matrix to produce sufficient energy necessary for physical activity. Myalgias, cramping, and 
chronic myoglobinuria are common skeletal muscle manifestations that occur in many pediatric patients with mitochondrial disease, when there is a large energy deficiency.

Exercise intolerance is often a prominent defining clinical feature of pediatric patients affected with mitochondrial dysfunction. Strength-type exercise results in an increase in muscular size, strength, and muscle fiber. In contrast, lower-intensity exercise extended over a longer duration promotes mitochondrial biogenesis. Dr Tarnopolsky suggested using endurance exercise as a potential therapeutic strategy, to increase size of the mitochondrial reticulum and halt the progress of muscular dystrophy often seen in patients affected by mitochondrial disease.

Dr Tarnopolsky referenced a clinical study of 20 patients diagnosed with primary mitochondrial disease (14 patients contained point mutations in the mitochondrial DNA; 6 patients contained genetic deletions of mitochondrial DNA), and compared their results with a control group of 16 healthy controls. Over a duration of 12 weeks, the study exposed participants to endurance exercise training (cycling training, 4 times per week). After training, patients who have primary mitochondrial disease experienced a $67 \%$ increase in citrate synthase activity as well as a significant improvement in $\mathrm{VO}_{2}$ peak, suggesting there are beneficial improvements for patients at the molecular and functional level. ${ }^{4}$

Another study demonstrated similar results. Patients who had a single genetic deletion participated in 14 weeks of cycle training followed by another 14 weeks of deconditioning. After the 14 weeks of deconditioning from the exercise, improvements in mitochondrial performance and exercise performance reverted to baseline values, demonstrating that clinical improvements are directly attributable to exercise therapy. ${ }^{5}$

Myosatellite cells are undifferentiated progenitor cells found in developing muscle cells and are essential in the muscle repair process. In infants, myosatellite or satellite cells comprise $20 \%$ of the total myonuclei in skeletal muscle tissue. Throughout the human life span, the percentage of muscular stem cells in adult muscular tissue will decrease to a level of approximately $3 \%$ or $4 \%$. Satellite cells are activated during exercise, where excess trauma is applied to muscular fibers, and are essential to muscle hypertrophy.

Patients with Kearns-Sayre Syndrome (KSS) contain a large-scale deletion in mitochondrial DNA, and a muscle biopsy will review cytochrome C oxidase (COX)-negative fibers. Dr Tarnopolsky referenced a study done by Dr Eric Schon who took muscle biopsies from patients with KSS and cultured them in vitro. The satellite cells in the culture all contained COX-positive muscle fibers, signifying that it had filtered out the large-scale genetic deletions, which shifted to a wild-type fiber. This technique was later termed mitochondrial DNA shifting. The theory underlying the mitochondrial DNA shifting proposes that trauma to affected muscle fibers stimulates wild-type satellite cells to proliferate and dilute the mutational burden within the myofibers and recruits mitochondrial DNA that does not contain the genetic deletion.

Dr Tarnopolsky led a study of 8 patients with chronic progressive external ophthalmoplegia who followed a standard weight-lifting protocol to assess the effectiveness of mitochondrial DNA shifting. During the study, the patients underwent 12 weeks (3 times per week) of 
endurance exercise training, including bilateral leg extension/flexion with an additional leg press. Genetic testing after the training regimen demonstrated a significant reduction in the proportion of deletions within the mitochondrial DNA, which provides additional support to the probability of mitochondrial DNA shifting becoming a new therapeutic strategy for patients with mitochondrial disease who are suffering from abnormal myopathies. Dr Tarnopolsky indicated that the effectiveness of this technique largely relies on where the genetic deletion is located in the genome, because mitochondrial DNA will only rescue deletions involved in DNA maintenance, such as polymerase gamma mutations.

There has been much discussion on the effects of mitochondrial dysfunction on aging. Researchers tested this in a mouse model and discovered that $P O L G$ mutated mice live approximately half as long as a wild-type mouse, and their aging phenotype is accelerated. The mutation is a specific point mutation of polymerase gamma-1 (D257A-POLG1) in the exonuclease domain of the POLG1 gene, in which an aspartate residue is converted into an alanine. This polypeptide conversion completely knocks out the polymerase's exonuclease ability to proofread its genetic information. In the D257A-POLG1 mutator mouse, an increase in oxidative stress and inflammation as well as a decrease in telomere length were detected, which are both classic features involved in the pathology of aging.

Dr Tarnopolsky used the D257A-POLG1 mutator mouse model to validate the benefits that endurance exercise has on attenuating the effects of the aging phenotype. The study consisted of 3 experimental groups. There were 2 treatment groups, POLG1-Sedentary group, which included all mutated mice that were sedentary throughout the 5 months of the study, a POLG1-Endurance group, which included mice with the $P O L G$ mutation that were exposed to endurance exercise (running) 3 times a week on a treadmill, and a control of wild-type healthy mice.

After 5 months, muscle biopsies were taken from each test and control group. In the muscle biopsies of POLG1-Endurance group, an increase in PGC-1a signaling was detected in the muscle tissue. PGC-1a is a chemical signal that will localize toward the mitochondria from the nucleus and stimulate mitochondrial biogenesis. In the POLG1-Sedentary group, PGC-1a remains in the nucleus, where it lies dormant until it is elicited by exercise. Through next-generation sequencing, Dr Tarnopolsky demonstrated that the POLG1Endurance group mice also contained a lower level of point mutations.

Endurance exercise was also shown to provide systemic effects to various organ systems. Dr Tarnopolsky compared the COX activity of both experimental groups and compared it with the wild-type control. In all tested tissues (heart, liver, skeletal muscle, and gonad) there was a statistical difference between the PolG-SED and the other 2 treatment groups. This finding suggests there may be a substrate promoted by physical activity that contains hormone-like effects on distant organ tissues. Dr Tarnopolsky labeled them "exerkines."

Dr Tarnopolsky mentioned that if specific excreted proteins could be identified, novel therapeutic options could be developed. A few of the protein targets tested included interleukin 6 and -15 , eotaxin, and VEGF. In a study of 100 people, serum was extracted from each participant at various times after physical exercise, and then added to cell culture 
to at the point at which mitochondrial biogenesis was stimulated. An increase in PGC-1a occurred quickly after exercise, particularly in athletic individuals, stimulating mitochondrial biogenesis in a cell culture of fibroblast. The finding was represented in an increase of mitochondrial copy number. This procedure was then recreated in a mouse model, with similar results.

The next phase of Dr Tarnopolsky's study sought to determine the specific protein agent that was stimulating the increase in mitochondrial number. The protein of interest was narrowed to be either interleukin-15 or tumor necrosis factor-beta (TNF $\beta$ ). After knocking out the gene for TNF $\beta$, there was still a stimulatory event, signifying that the stimulatory protein must be interleukin-15. Once the interleukin- 15 protein was inhibited with an antibody, the effects usually attributable to exercise were blocked, signifying the probability that interleukin- 15 is the protein of interest.

Translating this into a novel therapy, Dr Tarnopolsky compared 2 groups of mice. One cohort was exercised regularly for month and another group was sedentary but received interleukin-15 in a tail vein injection every day for 33 days. Injection of interleukin-15 produced similar results to treatment with physical exercise. Grip strength, muscle hypertrophy, and oral glucose tolerance were recapitulated in the otherwise sedentary mice, demonstrating the possible benefits injectable interleukin- 15 could bring for patients with mitochondrial disease who are not physically able to participate in physical exercise.

The final protein identified as of possible benefit to patients affected with mitochondrial disease is METRNL, a glial cell differentiation regulator-like protein. Various microarrays have shown this protein is secreted after both endurance and resistance exercise. METRNL appears to convert adipose tissue to brown adipose tissue, which contains increased mitochondrial content. This conversion of adipose tissue is usually associated with individuals with lower body weight and improved insulin sensitivity. The effects are not as pronounced as those associated with physical exercise, however. In a functional assay testing oral glucose tolerance, METRNL was shown to improve dysglycemia. The combination of interleukin-15 with METRNL was very effective in improving muscle strength, suggesting the 2 proteins could become very beneficial therapeutics for mitochondrial medicine in the future.

\section{Clinical Trials: Edison Pharmaceuticals, EPI-743}

Greg Enns, PhD, Stanford University School of Medicine, Palo Alto, California

A PubMed review revealed that only 75 of 140,000 cited articles dealing with mitochondria included clinical trials. Unfortunately, only 10 of those trials focused on genetic mitochondrial diseases, underscoring the fact that few studies have focused on evaluating potential treatments for these rare diseases.

Alpha-trocotrienol quinone (ATQ3), also known as EPI-743, is a quinone with a similar structure as coenzyme Q. Its similar chemical structure and properties make it an excellent electron transporter in various important biochemical processes. EPI-743 is currently being developed as a potential pharmaceutical compound to alleviate the severity of disease in patients affected with a mitochondrial dysfunction. 
In a simple cellular assay, Dr Enns applied buthionine sulphoximine (BSO), an inhibitor of glutathione synthase, on a culture of human fibroblast. With a decreased amount of glutathione, the cells remained under heavy oxidative stress. Introducing EPI-743 into the culture demonstrated its capacity to transport electrons in culture and decrease the oxidative stress experienced by the cell culture. EPI-743 may play an important role in reducing glutathione generation. Because of its size, it fits in the active site of nicotinamide adenine dinucleotide phosphate (NADPH) oxidoreductase (NQ01), a critical enzyme involved in alleviating intracellular oxidative stress. A current theory proposes that EPI-743 is associated with regeneration of reduced glutathione by providing a substitute for glutathione reductase.

Dr Enns performed various imaging studies viewing uptake of hexamethyl propylene amine oxime (HMPAO), which will detect how well mitochondria in various tissue samples are functioning in vivo. In an initial study, Dr Enns screened hexamethyl propylene amine oxime uptake in kidney tissue of mice. It was shown that as mice age, the signal for hexamethyl propylene amine oxime weakens, suggesting that mitochondrial efficiency also decreases with aging. After treating a cohort of mice with EPI-743, uptake of hexamethyl propylene amine oxime increased, along with improved peripheral glutathione concentrations.

A teenage patient affected with Friedreich ataxia at baseline demonstrated a 75\% decrease in uptake of hexamethyl propylene amine oxime in the cerebellar, right caudate, and medullary regions compared with wild-type mice. The decreased uptake of hexamethyl propylene amine oxime indicated where disease is specifically targeted in individual patients. Another patient was treated with EPI-743 on a compassionate basis, demonstrating an effective response within a 100-day trial period. Imaging revealed an apparent reduction in white matter disease, particularly in the medullary region and cerebellum.

A novel measure to differentiate disease severity in patients with mitochondrial disease has been developed recently and used in various clinical trials. The Newcastle Pediatric Mitochondrial Disease Scale consists of 4 sections. Sections I to III review the clinical assessment of each patient and section IV measures quality of life. In a recent study conducted by Dr Gregory Enns the scale was used to assess a cohort of more than 100 patients with a wide variety of mitochondrial dysfunctions, at various treatment sites around the globe. After treatment with EPI-743, most scores decreased, indicating a decreased burden of disease.

An additional study that assessed Newcastle Pediatric Mitochondrial Disease Scale scores and glutathione status showed a positive clinical outcome for most patients treated with EPI-743. The study measured glutathione at baseline as well as after EPI-743 treatment. Prior to treatment, levels of glutathione were significantly lower $(P>.01)$ than those noted in healthy controls. After pharmaceutical treatment with oral EPI-743, oxidized glutathione levels normalized, indicating an improved oxidative condition. This study demonstrated that glutathione could act as an important biomarker for oxidative stress in patients and serve as an indicator of mitochondrial disease in future therapeutic trials. 
In another study, 4 of 5 Leber hereditary optic neuropathy (LHON) patients treated with EPI-743 showed improved visual acuity. One particular participant started the study with a baseline measurement of 20/400 in the right eye and 20/200 in the left. After treatment, vision greatly improved, to $20 / 40$ and $20 / 25$, respectively.

Dr Enns concluded by sharing an overview of a developing study, in which he has enrolled 30 patients with mitochondrial disease. Prospective participants were screened for various inclusion criteria, such as moderate disease based on the Newcastle Pediatric Mitochondrial Disease Scale score, and documented evidence of disease progression (such as an MRI) that confirms diagnosis of Leigh syndrome. The cohort was screened using a list of exclusion criteria as well. In this double-blind, randomized, placebo-controlled study, half the patients will receive either placebo or EPI-743 for the first 6 months. The following 6 months, all 30 patients will receive EPI-743, but in varying doses. Treatment effectiveness, the chief outcome measure, will be assessed using the scale (sections I to III).

The number of clinical trials to treat mitochondrial disease is increasing, as well as the number of potential pharmaceutical agents being studied. Dr Enns mentioned Bendavia is another treatment supplement being assessed to treat these patients. The growing interest in clinical trials of treatment measures offers hope that patients suffering from mitochondrial disease will have new options to manage their disease.

\section{Occupational, Environmental, and Therapeutically Acquired Mitochondrial Disorders}

Kendall Wallace, PhD, University of Minnesota School of Medicine, Duluth, Minnesota

Dr Wallace reminded the audience of the powerful effects the environment can have on patients suffering from mitochondrial disease. He prefaced his discussion by stating that, to date, researchers have largely focused on understanding the genetic etiology of mitochondrial disease. In comparison, not much has been studied with respect to occupational or environmental exposures that could promote development of disease. Many environmental features, such as particular pharmaceutical agents, can provoke development of mitochondrial defects within patients who would be diagnosed with "secondary mitochondrial disease."

The electron transport chain is a major target for external agents to initiate disease development. Because the electron transport chain is composed of more than 100 subunits encoded by both the nuclear and mitochondrial genome, altering any one of the essential subunits could result in development of disease pathology. Dr Wallace discussed the basic machinery of the electron transport chain and how environmental as well as pharmaceutical agents can alter the molecular mechanisms of the mitochondria to produce inadequate ATP.

The Krebs cycle produces reducing agents into the mitochondria, which ultimately arrive at complex I and donate an electron to initiate the electron transport chain. As the electrons are passed through each complex, protons are pumped across the inner mitochondrial membrane into the inner membrane space, creating an electrochemical gradient that ultimately will power the ATPase located in complex V. The transmembrane potential created across the inner mitochondrial membrane is approximately 180 millivolts $(\mathrm{mV})$ difference. 
Maintaining this membrane potential is crucial to proper mitochondrial function. Without sufficient electrochemical potential energy, there would not be enough Gibbs free energy to drive the phosphorylation process of complex $\mathrm{V}$.

Unfortunately, multiple chemical agents are capable of disturbing this transmembrane potential. The active pumping of protons into the inner membrane space creates a negative charge along the inner portion of the inner mitochondrial membrane, which often attracts divalent cations to cross through calcium channels. The accumulation of divalent metals replaces already existing cofactors, and they bind preferentially to matrix proteins, hindering the redox cycle. The phospholipid bilayer potentially attracts lipophilic toxins that can pass freely through the membrane and affect the matrix biochemical processes. In addition, cytochrome P450 grants the mitochondria an innate drug-metabolizing ability.

Environmental agents such as benzopyrene can be converted to a toxic epoxide, which will interact negatively with the mitochondrial DNA.

Dr Wallace displayed a list of pharmaceutical agents that interfere with the various complexes of the electron transport chain (complexes I to V). By blocking the electron transport chain, NADH is not being oxidized and oxygen is not being reduced, creating cytoxic hypoxia-where there is sufficient oxygen, but ATP is not being catalyzed. 2,4Dinitrophenol is a protonophoric uncoupler, which carries a proton in the inner membrane space and diffuses across the lipophilic membrane to the alkaline inner matrix, where it donates the proton in the mitochondrial matrix. Working against the proton pumps, 2,4dinitrophenol dissipates the $180 \mathrm{mV}$ transmembrane potential established by the electron transport chain. With insufficient ATP, energy consumption adapts to using NADH and the excess energy created along the electron transport chain is then converted into heat (the metabolic profile clinically presents itself as a fever). Nonsteroidal anti-inflammatory drugs, such as naproxen, have been associated with provoking such disease pathology in patients with mitochondrial disease.

Molecular mechanisms similar to those seen in protonophoric uncouplers are seen in electrophoric uncouplers as well. Many electrophoric uncouplers are positively charged cations $\left(\mathrm{PB}^{+}, \mathrm{Ca}^{2+}\right)$, which also reduce transmembrane potential, causing cells to use glycogen and adipose stores, creating a wasting-like syndrome.

Chemical compounds also can target the redox cycle involved in the electron transport chain. Quinone-like compounds such as gentamicin and benzoquinone have been previously shown to steal electrons from the electron transport chain and produce unstable free radical intermediates. Once reduced, the compound oxidizes into its parent compound and donates the radical to molecular oxygen, creating an accumulation of reactive oxidative species, which can interfere with and disrupt processing of cellular genetic information.

Dr Wallace also shared a list of molecular targets that could promote disease development. Antiviral drugs primarily have been shown to inhibit polymerase gamma from replicating, and transcribing mitochondrial DNA, resulting in petite mitochondrion-deficient cells. Many compounds designed as antibacterial agents inhibit mitochondrial protein translation. Antibiotics such as chloramphenicol and tetracycline will block the minor groove between 
the messenger RNA and the polyribosome to inhibit not only bacterial protein translation but also mitochondrial protein synthesis. The inhibition of mitochondrial translation provides further support to the prosymbiotic theory that highlights the mitochondria's prokaryotic origins.

Dr Wallace closed by reiterating a point made by Dr Robert Naviaux: that $95 \%$ of mitochondrial disease is of nongenetic etiology. The statement further underlined the importance of being aware of the environment that surrounds the patient, and the role it can play in disease prognosis.

\section{Question and Answer (Session III)}

Dr Maria: How do we know that environmental exposure actually produces a functional effect? It's true in a mouse model in a laboratory setting that one could take each of these compounds and have a target and potentially produce an effect, but isn't the therapeutic window, if you will, quite wide for environmental effects? What objective evidence is there for functional mitochondrial limitation from exposure to environment, to drugs? What's the evidence?

Dr Wallace: That's a very good question.

The environmental exposures, the thresholds to manifestation of the disease, are probably no different than what was discussed for the genetic threshold. There's an overabundance of excess capacity. It's also been called of mitochondrial bioenergetics potential or capacity. You can inhibit a certain degree of it, depending upon the tissue in the energetic state, before you'll see any manifestation of a tissue in the energetic state, before you'll see any manifestation of a tissue organopathy of any type of disease injury. It varies between tissues.

There was a study done 15 years ago with an isolated hepatocyte. The HepG2, we're all very familiar with those. They're not very representative of human primary hepatocyte. In that study it was necessary to inhibit $70 \%$ of electron transport before you saw any indication of hepatotoxicity. I'll remind you that a liver cell just sitting in a Petri dish with a lot of substrates probably doesn't have a high metabolic demand. It's not going to start digging into its reserve capacities. If you take an isolated cardiomyocyte and you put it in a dish and you expose it to something that interferes with mitochondrial metabolism, you will start seeing that evidence of decreased contractility, decreased contraction rate, and that at lower capacities. Then if you start stimulating that cell to beat, you'll start getting much closer to the maximum potential, maximum capacity of that cell.

I'm talking around your question. I realize that, but what you see depends on the conditions of that individual, sedentary versus stress.

Dr Hirano: Maybe I can give you a concrete example. The drug you mentioned, linezolid, is used in the ICUs frequently for methicillin-resistant staph aureus. It's approved for use for 2 weeks. But if you use it for more than 2 weeks in patients, some of them will develop optic neuropathy, peripheral neuropathy, lactic acidosis, all typical mitochondrial features. If you use that same drug in tissue culture, you'll 
see shutdown of mitochondrial protein synthesis in about a week in our hands at the concentrations that are typically seen in the patients.

What happens in the tissue culture doesn't translate exactly into what happens in the patients. There was a delayed effect in patients, but it's clearly very similar. There is a difference between what goes on in the lab and what goes on clinically, but some of these will translate into real toxicities.

Dr Wallace: Just a follow-up on that with our experience with propofol because you saw that on the list; certainly that comes up at every mitochondrial meeting. Should we be using propofol when we're doing LP or a muscle biopsy in someone suspected with mitochondrial disease? We've used propofol in over 1000 children for muscle biopsies in LPs. We usually combine them together so it can take up to 12 minutes. We haven't had a single incidence of any adverse effects whatsoever, but obviously, as is the case with other drugs, when you're using propofol in the ICU for long periods for which it was not intended to be used, all bets are off and then you can start running into toxicity. I would caution people looking at this list and saying these are not good drugs. You should never use them. Some of the fibric acid derivatives are PPAR agonists and PB delta agonists, which can actually improve mitochondrial function when you grow cells in them for a long period of time. That's my only caveat to that.

Audience: Are any of the compounds relatively or absolutely contraindicated in our patients with mutations with mitochondrial disease?

Dr Tarnopolsky: I think valproic acid in the patient with known POLG mutation.

Dr Tarnopolsky: Valproic acid in a patient with a known $P O L G$ mutation adds to the contraindication.

Dr Enns: Absolutely.

Audience: Would the panel comment about phenytoin? It's been listed up there and it's been listed for years, now being less used hopefully. Did we see more problems with mitochondrial conditions then as opposed to now?

Dr Wallace: Once again, I derived this list. I just borrowed it from Dr Will. The fact that it's on the list means that it was tested and it was shown at sufficiently high concentrations that it will interfere with mitochondrial function. I'm going to defer to the clinicians as far as any evidence for types of mitochondrial disease with phenytoin.

Dr Tarnopolsky: I think practically the most important thing in a child is to make sure that they're not having seizures. Other than valproic acid in a patient with POLG mutation, you use what works. All bets are off when you're dealing with mitochondria, as it is with any other type of complex seizure disorder. Certainly, Keppra is well tolerated. Is there an absolute contraindication? If Dilantin worked well, I see no reason why you shouldn't continue with it. We've certainly had patients with mitochondrial disease who have been on it for years to decades without problems. 
Audience: This is just more of a comment about improving how we communicate medical principles to our students. What we're hearing today is that it's the dynamics and the pacing and the response over time in disease that gives us insight into mechanism of action. Medical students are frequently taught from snapshots that a disease presents in total all at once. Frequently the deepest insights of pathogenesis emerge or are encoded in the timing of the response after being exposed to an agent. Teaching in kind of video, or how classically medicine was taught as an apprenticeship, is something that I think is an essential part and should be actively reincorporated into how we train the next generation of neurologists and biochemical geneticists. I thank everybody for a kind of highlighting that importance of the dynamic aspect of the disease and what we're understanding.

Dr Tarnopolsky: Can I just make a comment? Not just toxicity, but we have to remember deficiencies as clinicians. I'll just give you several examples in our clinic. Thirteen percent of the patients when we measured them had vitamin $\mathrm{B}_{12}$ deficiency, which is probably one of the least-tolerated deficiencies in the mitochondrial disease patient. Four percent have red blood cell folate deficiency; upwards of $10 \%$ of children had cerebral folate deficiency. Something struck me just 2 days ago. We had a young girl who had MELAS syndrome. She was stable for quite some time on the mitochondrial cocktail doing exercise. Everything was good. She started losing hair, gaining weight, and unfortunately didn't come back to see me for about 6 months. She was profoundly hypothyroid. We know that thyroxine is a very potent stimulator of mitochondrial biogenesis. Clearly I think the thing we must not forget is that deficiencies can make our patients worse and don't forget the common things that are easy to identify and rectify.

Dr Wallace: The other thing I'd like to add to that, and I didn't have time in my talk, is that when you have a person with longstanding mitochondrial disease, they undergo what I call metabolic remodeling through nuclear receptors such as PPARalpha and PPAR-gamma. They start expressing genes that they don't normally express in those tissues. In my hands, anyhow, with adverse environmental exposures they upregulate the mitochondrial proliferation, tying to increase the mitochondrial numbers.

I like to look at it as a compensatory response to try to replenish the lost bioenergetic capacity they have as a result of that exposure. When they walk into your office, their metabolic metabolomics may be quite different than that of an unexposed or a nongenetically modified individual.

Audience: Compensatory mechanisms upregulate the mitochondrial DNA through the PGC-1 alpha pathway. At the same time, it looks like there's a purging or a lowering of mutation rates. Is that a dilution effect? I know it's been speculated, especially following Richard's talk. Are we seeing evidence of upregulation of mitophagy events?

Dr Tarnopolsky: Certainly there's a lot I didn't talk about in the POLG mice, but if we take them as an example, exercise is a forme fruste of a biological filter. What happens is that the mitochondria that have low membrane potential essentially get 
targeted for removal from the cell. It's almost biological fitness in the cell. The exercise is a stress test which forces them through this biologic filter. We do see increase in mitophagy in our POLG mice who are exercised to remove bad mitochondria.

There clearly is evidence of shifting of the stem cells, and the sporadic disorders have low mutational burden. I think that's one issue. We have a paper that we've submitted recently showing that p53 can migrate. Ayesha Saleem working with David Hood has shown this too. It can go into the mitochondria. We've shown that it has a unique effect to actually repair some of the point mutations, so it can partially compensate for a dysfunctional POLG. I think the benefit of exercise is so multipotent and very potent that it's difficult to recapitulate.

Even though we're showing some benefits with interleukin-15 in Metronil, I think that it really is nature's gold standard in terms of multisystemic mitochondrial fitness. There are innumerable pathways. That's why to some extent it's a tough thing to study. The pure molecular biologist says it's dirty because it's not just one mechanism. But that's the beauty of Mother Nature, I think, is that there are so many mechanisms. Cells don't die by one mechanism. Every cell. I don't care if it's Friedreich's ataxia or mitochondrial disease, excitotoxicity, oxidative stress, mitochondrial dysfunction. So many factors that a singletargeted therapy or a single mechanism to explain pathophysiology is going to be incomplete.

\section{Executive Summary of the Day}

\section{Dr Bruce Cohen and Dr William Copeland}

Session I covered the clinical aspects associated with mitochondrial disease. Dr Schon provided a short introduction to mitochondrial structure and the importance of the double membrane in the production of ATP. In addition to energy production, the mitochondria are responsible for intracellular signaling for cellular processes such as apoptosis. When the mitochondrial machinery is not performing properly, free radicals that can further damage important cellular components accumulate, which can lead to various cancers and degenerative diseases. The mitochondrial DNA also contain various genetic loci responsible for producing critical components of the human mitochondria. Disruption of any of the various genes products from the mitochondrial genome can disrupt the highly conserved mitochondrial dynamics. Dr Cohen discussed the difficulties of defining mitochondrial disease, as patients with mitochondrial dysfunction can present in clinic with a multitude of comorbidities that make it difficult at times to provide a concrete diagnosis. Dr Chinnery introduced the concept of heteroplasmy, underlying the reality that varying levels of disease representation are reflected within varying genotypes. Penetrance of disease of a particular genotype in mitochondrial disorders is largely determined by the mutation threshold in which disease presentation will occur. Dr Naviaux completed the first session of the symposium by separating mitochondrial disease into primary and secondary mitochondrial disorders.

Session II covered the molecular mechanism associated with mitochondrial disease. Dr Copeland discussed how defective mitochondrial DNA replicative machinery can play a 
large role in mitochondrial disease pathology. Subunits such as polymerase gamma exonuclease transcribed by the $P O L G 2$ gene is essential for polymerase gamma's proofreading ability and, when altered, can lead to embryonic lethal mutations. Other components of polymerase gamma were discussed in detail in addition to the essential role they play in propagating the mitochondria's genetic information. Dr Youle continued the second session of the symposium with a presentation highlighting mitochondrial dynamics in relation to neurologic diseases such as Parkinson's. Disruption of the mitochondrial quality control pathway was considered in Dr Youle's presentation to be a major determinant in the development of adult Parkinsonian disorders. Dr Mootha finished the second session, with an in-depth discussion of a systems biology approach to mitochondrial research. Dr Mootha contributed a lot of new information in regard to how sequencing technology has recently led to the discovery of essential mitochondrial genes such as MPV17, which is crucial in mitochondrial DNA maintenance. In his presentation, Dr Mootha described taking advantage of a computational method called exome sequencing to identify more than 1300 nuclear genes involved with the mitochondrial genome.

Session III covered the translational science and clinical frontiers of mitochondrial disease. Dr Hirano, of Columbia University, opened the session by discussing a novel therapy of shifting the heteroplasmy under an established disease threshold using a restriction enzyme to excise mutated versions of genetic information from the human genome. Dr Tarnopolsky continued the final session by discussing the various benefits that clinical trials using endurance and resistance exercise have had on patient and mouse models. A large portion of Dr Tarnopolsky's presentation focused on exercise intolerance in patients affected with a mitochondrial syndrome. Dr Tarnopolsky mentioned a specific clinical trial in which a cohort of patients was exposed to 6 months of exercise training. Results from the clinical trial demonstrated a significant decrease in the number of genetic deletions, which correlates to better disease management. In a mouse study, Dr Tarnopolsky monitored various levels of PGC-1a during endurance training therapy. The response to treatment showed an increase in PGC-1a in various biological tissues as well as an increase in Cox activity shown in muscle biopsies. From this, the overall mitochondrial morphology and physiology improved in comparison with the sedentary control. Dr Enns touched on another novel therapy, EPI-743, which is currently in clinical trial to test whether it improves mitochondrial function in a cohort of patients suffering from various mitochondrial syndromes. Dr Wallace ended the symposium with a discussion on the effects of environmental toxins, such as pharmaceutical agents, on the development of mitochondrial disease pathology. Dr Wallace mentioned that many targets for disease initiation don't simply include the electron transport chain, but also other molecular components throughout the biochemical pathway that are involved in energy production. Dr Wallace emphasized that acquiring disease is not just genetic in etiology, but that clinicians need to be wary of environmental agents that could potentially cause harm to the human mitochondria.

\section{Future Directions Panel Discussion}

Dr Wallace: We've had some really exciting insights in the last 8 hours. One insight is of course that in traditional medicine, we've tended to look at anatomy as 
the basis for diagnosing disease; hence, having all of the clinical subspecialties, which are anatomically based.

We've tended to think of genetics as quantified; hence, because of its Mendelian origins from the nuclear genome's perspectives. We now know that to be alive is to be adamant. As Newton said 450 years ago, no mass will move without energy or in his case, force. Therefore, the most important thing, as you've heard repeatedly, is the fact that we're energized. Therefore, there's I think a tremendous new era in medicine where we move away from this anatomical perspective on disease and start thinking holistically about the genetics of disease.

That of course then brings in, just as you've heard, another kind of genetics that is not quantified, but quantitative. This quantitative genetics is directly related to energy. We have this new and parallel view of biology and medicine. It will revolutionize the way we look at the diseases. That's because so many of the common complex diseases that we're asked to understand are in fact not easily understood by either the anatomical or the Mendelian genetic perspective; hence, complex diseases.

At the same time we have these philosophical changes, we're seeing technological changes. Previously of course, technology for medicine was based on anatomy. Of course, all of you got your stethoscope. That was your right of passage to become a physician. Then later you learned that you could have used the NMR machine. All of these were ways of talking about anatomy. It always struck me as interesting that the radiologists using various imaging techniques were often looking at energetic phenomenon, but talking about the results from an anatomical point of view. We now actually can reinterpret a lot of our anatomy from an energetic perspective.

At the same time, we also have an evolution of technology at the other side; as Dr Mootha mentioned, previously we thought of genetics from a Mendelian point of view and from mapping genes. Now we have next-generation sequencing where we simply look at nucleotides. We're going to see a lot of parallel advances that I think are going to completely restructure medicine and hopefully also medical education to the benefit of these patients with complex disease.

I'm really privileged then to be able to be here with this distinguished panel. I'd like each of them to introduce themselves and tell a little bit about what they think the future might be. Hopefully, we can get your insights as well. I'd like to start in alphabetical order because women are first here.

Dr Goldstein: Thank you. I'd like to thank the organizers for inviting me to be on the panel. I'm at Children's Hospital of Pittsburgh. We are a NAMDC site. I'm actively recruiting patients. I see a lot of mitochondrial disease patients there. I'm also on the UMDF Board of Trustees. I'm actively working on developing common data elements with NIMDS.

I think as far as future directions go, one of the things that I would like to see developed are clinically relevant endpoints that we can all use, whether we're designing natural history studies or we're developing clinical trials. One of the things that Patrick Chinnery 
discovered when he did this Cochrane Review and then published the new paper he mentioned on treatments, "No Time to Drop Our Standards" I believe is the title. You can't compare so many of the clinical trials that have been published because they did not use the same clinical endpoints and it was a very small sample size. My ultimate goal would be that we have clinical trials, that anyone who has mitochondrial disease can get a diagnosis without too much difficulty. Then they can immediately be enrolled in a clinical trial and we can compare those trials with similar endpoints.

Dr Gropman: Hi. Thanks for the opportunity to be on the panel. I just wanted to give a little bit of perspective of how I got involved in what the past was before I talk about the future. First of all, I'm a child neurologist, obviously. I'm also trained in clinical and biochemical genetics. I remember attending my first mitochondrial meeting in Indianapolis and just bringing back information to my department. At the time, not much was known, except the fact that these were complex disorders and they were complicated to diagnose. That still holds true. We didn't really have a lot of genetic tools at our disposal.

Most patients had biopsies and that was pretty much all we did. Then over the years we saw with the advancement of molecular genetics that transferred into the mitochondrial field as well. We were able to take advantage of primarily mitochondrial DNA-encoded genes. Then only recently, over the last decade or so, we've had panels for nuclear genes. I think some of the things that we need to keep in mind as the technology goes forward at a rapid pace is the important of our clinical exams and phenotyping the patients, not only in terms of deciding who should get mito exome versus one of the panels or a single gene if we know exactly what the disorder is, but also after the results come back, it's really important to determine if what you get really fits with the presentation of the patient, the family, taking the whole pedigree, if you will, into consideration, because there may be all of those symptomatic individuals in the family who you didn't really think were harboring a mitochondrial disorder.

Then looking at biomarkers. Part of what I do in addition to seeing patients is I also do research in neuroimaging and inborn errors in metabolism. I work in the urea cycle world as well, OTC deficiency, which is in the mitochondria. That enzyme is one of the proximal disorders that is mitochondrial enzyme. Looking for ways that we can apply noninvasive measures to look for the earlier stages of disease if that can inform us about treatment, and then being able to use those as endpoints for some of the clinical trials as they're going forward.

Dr Haas: I'm a pediatric neurologist and I run neurometabolic clinics for both children and adults. I do have some internal medicine training to justify that. I've really just been thinking about what patients want from me when they come to see us in the clinic.

There really are 3 different areas. One obviously is a diagnosis. A lot of people, and children, come with a question as to whether they have mitochondrial disease. Another is what is going to happen to them? What's the course and the prognosis for the disease that they have? The third of course is what treatments do we have available? 
I think as we move forward we have to address all of these issues. You've heard a lot of information today about various forward movements in all of these areas. I think in terms of diagnosis you've heard a lot about genomics. Certainly that's becoming a day-to-day issue for all of us, I think, with the availability of next-generation sequencing. The problem certainly in my part of the world in California is that the insurance companies are not allowing us to do this. There are a number of roadblocks as we move forward that are really societal in terms of allowing us to do the testing that we need to do on our patients.

We are frequently asked if we're going to justify a test, whether it's going to make an effect on the treatment of the patient. That's sometimes a difficult thing to certify, that we're actually going to be able to help, because that's another area of course with mitochondrial disease. We're really just at the beginning of hopefully having some effective treatments.

I chair a clinical trials committee for the UMDF. One thing that we're going to be focusing on in the very near future are some protocols that will help us do clinical trials in this heterogeneous population, both clinically and in terms of molecular defects.

We certainly need biomarkers. There are some new biomarkers coming along that we're exploring: FGF 21, fibroblast growth factor 21 as a marker of muscle mitochondrial disease is one. You've heard about glutathione, another one. These are not readily available in the clinic yet, but moving forward I think they're going to help us quite a lot.

We need to really focus I think on the final common pathway of these multiple causes of mitochondrial disease. One group of patients that are really good to start with I think in terms of designing clinical trials are those with muscle disease, because we need to have hard numbers and actually show improvement in parameters that are useful to the patients and to the FDA.

I think that whole effort is moving forward. There are a number of promising compounds on the horizon. Quite a few biotech companies have got agents that they're working on that look like they should be safe and helpful.

Then the final thing I just want to comment on is education, which is really important from the diagnosis and treatment perspective. We have a fellowship program in the North American Mitochondrial Disease Consortium, which is aiming to train admittedly a small, but hopefully important, number of clinicians in mitochondrial disease. It's a one-year training program where they rotate to different centers throughout the country.

Of course what we're doing today is also moving the field forward. We really need to bring knowledge of mitochondrial disease to all of the clinics that these patients turn up in and continue the effort to educate our colleagues. Thank you.

Dr Parikh: I'm the director of the Neurogenetics and Neurometabolism program at the Cleveland Clinic. I run the Mitochondrial Disease Clinic there. I'm also part of NAMDC, as well as on the Medical Advisory Board for the UMDF.

When I first started learning about mitochondrial disease, one of my teachers, Chuck Hopple, started out talking to me about Star Wars. I hope most of you in the audience have 
seen Star Wars. I actually asked my residents when I was giving a resident conference, and only half of the group raised their hands.

Let me tie this back in. Chuck Hopple started by saying Luke Skywalker, when he's learning about his powers and being a Jedi, he asks about the Force and he says, "What is the Force?" I forget who it was. I don't remember if it was Obi-Wan Kenobi or somebody else, but they answered that the force is an energy field created by the midi-chlorians in all living things.

Midi-chlorians reside within the cells of all living things. They are symbiotes with all living thing. Without them, life could not exist. The Jedi have learned how to listen to and coordinate the midi-chlorians. Midi-chlorians surround us, penetrate us, and bind the galaxy together.

Of course George Lucas was talking about mitochondria. We've kind of learned today that mitochondria really do permeate so many aspects of medicine. As a clinician I found that so very challenging. Do I worry about mitochondrial disease in every single patient I come across? I know there are some people here who would say yes, you should. But at the same time it really creates a dilemma because even if it's secondary mitochondrial dysfunction, I think families, patients, they don't always understand the distinction. They take the concept of mitochondrial dysfunction and translate it into primary mitochondrial disease, and they run with it.

As we have learned from some of the talks today and some of the work done by so many others, many of the patients that we end up seeing don't have primary mitochondrial disease. They in fact end up having another genetic or metabolic disorder. I tell my residents all the time that if you're at a mitochondrial disease clinic, most often the majority of what you're going to see is not mitochondrial disease. It's going to be a whole host of other conditions.

The reason I bring this up is because I'm supposed to talk a little bit about the future and what we see. But I think we can't forget that this is a past that needs to stay with us and follow us because it's important. I think as clinicians we need to be splitters, not just lumpers. I think for the research mind, lumping is fine. It helps kind of form new connections. As clinicians, the information we give our families, being able to say this is a primary mitochondrial disorder or this is not. Your prognosis is going to be different, your outcome is going to be different, and your treatment might be different. You don't need EPI-743. You need a different medication. I think that's just as important.

As far as the future goes, it's been very fun to grow up with all this explosion and knowledge that's happened over the last 10, 15 years. Being primarily a clinician I've been able to kind of learn all of this and take all of this new knowledge and use it.

The way we make a diagnosis is obviously changed and it's going to continue to change. Genomics is important, but I don't think we are done with biochemistry. Up until relatively recently it's always been mitochondrial disease, go get a muscle biopsy. That's obviously changed. We're going to now get a gene panel. Then we're going to get exome testing.

What we're learning by work done by Dr Mootha, as well as many others-Marnie Foggthe gene panel gives us a diagnosis $25 \%$ of the time. Exome is only giving us a diagnosis 
about $25 \%$ of the time. That still leaves us half our patients, well phenotyped or not, who don't have a diagnosis. Then we're going to be having to fall back on our biochemical knowledge and our biochemical means of making a diagnosis. I don't think it's completely left us. I think it's just changed the order that we end up pursuing this testing in.

As a small aside, I feel like when I was taught about getting a muscle biopsy I was always taught about histology, histopath, electron microscopy, electron transport chain enzymology. It's not that those tests aren't important, but histopathology, it turns out, in pediatric patients is often normal. Because of the lack of sensitivity of that test, it's not as important as some of the other testing that we could do.

I feel like the things that I learned after I left child neurology when I went into fellowship was that the muscle really is so useful for looking for a coenzyme Q10 deficiency because this is a primary mitochondrial disorder that is potentially treatable and modifiable. It can be used to better detect mitochondrial DNA deletions and depletions. This testing is much more accurate in muscle, as opposed to any other tissue thus far. Our ability to measure heteroplasmy is much more accurate in muscle.

I believe it was Dr Schon who was talking about the threshold effect and that you need a certain threshold before you have symptoms. However, if you only find the mutation in blood or even say in other tissues, you might actually only find a low-level heteroplasmy of a mutation. That does not necessarily mean that this patient is not going to be symptomatic or is symptomatic from this low level heteroplasmy, because the amount of heteroplasmy and burden of the mutation in deeper tissues might be so much higher. That's the other important reason that we still need muscle.

Then of course the last is to sort out these variants. We're going to find so many variants. There is going to be a lot of functional testing that needs to be done from the genomic side, but many of us don't always have access to lab facilities that allow for that. How else could we potentially prove that this variant is causing a functional defect in how the mitochondria work? Well, we have to fall back on our biochemical studies and proteomic studies. That's where the muscle biopsy hasn't become less important. Its importance has just shifted and changed. That's as far as diagnosis goes, the diagnostic methods we use.

The other challenges we've had in the field are that there has been a bit of a lack of consensus. Everybody has been doing things a little bit differently. There has always been a concern that you could go see $\operatorname{Dr} \mathrm{X}$ and you end up getting one diagnosis, and you'll go and see Dr Y and you'll get a different one, and Dr Z may or may not agree with one of those individuals.

I'm going to make a small pitch for the Mitochondrial Medicine Society. That is something that Dr Richard Haas as well as Dr Bob Naviaux started quite a while back, and helped many of us kind of join the society. We have expanded and grown. The reason I talk about the Mitochondrial Medicine Society is because we just finished a project where we started looking at how people are practicing mitochondrial medicine across North America. It was partly to confirm our fears that there really is too much variability in this field, and how people are practicing differs considerably. 
There are some similarities, fortunately, but there is a bigger concern that you really can go to 5 different centers and end up getting slightly different or 5 different diagnoses. I think as a clinician in the field, I don't know if I feel comfortable with that. I think this is something that in the future we need to change.

Again, the Mitochondrial Medicine Society, as well as a group of other individuals, are hoping to change that. We're in the process of trying to create consensus criteria not to establish diagnosis, but you've got a patient with potential mitochondrial disease in your clinic. What should we do next? What is the right set of next steps to take? How should you zig or zag depending on your results? We'll see how that turns out, but that's one of our goals for the future.

I only have 3 other small points. This is a relatively rare disorder and because of it there needs to be collaboration. It is wonderful that through Michio Hirano and Columbia we have NAMDC. It's the first time the United States or North America has had such collaboration. I think it is one of the few ways that we are going to actually get a large number of patients that we can study. Otherwise, we're all left with our own 5 or 10 patients with condition Y and 5 or 10 with condition $\mathrm{C}$ and not enough that we can actually build conclusions on. The hope is that maybe this can also end up becoming kind of an international collaboration. The Mitochondrial Medicine Society hopes to participate in that and work on that.

Other hopes for the future. A little bit of what Andrea and Dr Haas talked about, that it is nice to have treatment options in the forefront. It is nice to have double-blinded randomized trials happening for mitochondrial disease. We all have our fingers crossed and hope that these trials pan out and show us something hopeful. It would be very nice to have better biomarkers, to be able to follow and track this disease, and be able to offer our families prognosis and help them with determined outcomes besides just offering genetic counseling.

All in all, my families ask, "Why don't we have more for mitochondrial disease?" Usually I tell them to kind of take a step back and say you have to see that it's a very young field of medicine. We only identified the first mitochondrial disease in the 1960s, and most of this research has really exploded over the last 20 years. When you compare things like diabetes, which has been known about since BC times, Alzheimer's for 200 years, febrile seizures for about 100 to 150 years, we still have a lot of catching up to do. When you then compare and contrast how much we've advanced in such a short amount of time, I think things look a lot more hopeful than they would otherwise. That's it for me.

Dr Saneto: I'm sort of in the trenches. My other hat is epilepsy. I guess most of us in here treat patients who have epilepsy. Being an epileptologist, I think I'm one of the far left people as far as being a splitter. I just really encourage all of you to send your patients to NAMDC so we can learn natural histories, so I can tease out the difference between a whole gamma 467 homozygous mutation who's 15 in status versus a 467-28QX truncation heterozygote that I'm just going to be at the hospital for the next 2 weeks straight.

We need better drugs for epilepsy, but in particular we need better drugs for mitochondrial patients who have epilepsy. I'm really on one side of the fence because that's what I do, but 
$40 \%$ of my patients who have mitochondrial disease have intractable epilepsy. That's above the $30 \%$ if you really believe Brody's work. Most of my patients have intractable epilepsy. I have to mix and match different seizure medications in hopes of trying to control their seizures somewhat to give them a better quality of life. Of my infantile spasm patients, 5\% of them have mitochondrial disease. ACTH works, but after that the options are limited.

We need better tools to diagnose early. We need to get like the cystic fibrosis people to find that one particular mutation that responds to a drug. We would probably need 100 of those because there are 200 different mitochondrial diseases. If you're young and you have mitochondrial disease, your chances of having epilepsy go way up.

In the end, I think I'll just reiterate 2 things, one that Bob Naviaux said. In the end, metabolics trump genes because it's pathways. Genes are responsible for pathways, but it's the interaction between those genes that makes the big difference.

When I was an intern at the Cleveland Clinic, I remember I used to keep these $3 \times 5$ cards with all the symptoms of a particular disease. If you ask me, I will tell you exactly what the symptoms would be for a particular disease. They're all alphabetical because I got them out of NHL, so you know patient studies. I went into a room and this patient had all 6 of my diagnostic criteria for a particular disorder, and they didn't have the disorder. I remember my attending, who happened to be Bruce Cohen, looked at me and goes, "You know Russ? I think we're going to have to be doctors on this one." That's so true in mitochondrial disease.

Not everything presents easily and diagnosable. I guess my future would be earlier diagnosis, a tethering and teasing out of the different types of mitochondria disease, better epilepsy medications not only for my mitochondrial but for all my epilepsy patients, and good collaboration among all of us. That's what we need.

Dr Wallace: Thank you very much. One of the themes that we've heard repeatedly is the complexity of mitochondrial disease and the necessity for communication, because we have to come up with some method for parsing out these different diseases and developing ways that we can both diagnose and treat them.

Developing better data systems, data analysis systems, and data integration will be very, very important. Certainly Dr Mootha mentioned some of the efforts to coordinate internationally information about whole-exome sequencing so that rare patients could be brought together and then you could confirm the molecular diagnosis.

As a very brief set of comments, Dr Deb Hirtz would like to mention a few points about data analysis and common data elements for maintaining these kinds of databases.

Dr Hirtz: Thank you very much. I know that these have been mentioned. I know that Dr Goldstein mentioned them and probably others have today, but I wanted to be a little bit more specific about what is happening with the mitochondrial disease common data elements.

As you might imagine, having common data elements is going to increase efficiency of data collection and especially of harmonization across different studies. It will facilitate the sharing of data. It also will facilitate the performance of clinical trials and studies. It will be 
much easier to start up by using a case report form and other instruments and will improve data quality, reduce study start-up time, and make sure that we see the same questions being asked and answered in the same format.

Here is the list of current common data elements that have been established and the ones that are in process. In red you see neuromuscular disorders. In particular, the congenital muscular dystrophy is just finishing up and they're working on myotonic dystrophy and facioscapulohumeral muscular dystrophy as well right now.

The mitochondrial disorders, CDEs, are just in process. There will be a number of things available on the website once these are done. In particular, there will be data dictionaries, which should be very helpful, as well as some of the other forms that would be available for any studies in mitochondrial disorders.

Because we really expect these to be used in all future applications for clinical research and in all future studies for clinical research, it's very important to get in on the ground floor and make sure that they're done correctly and according to the best standards in the field.

What we would very much like is to have some more volunteers for the mitochondrial CDE project. It is hopefully underway now, but the panel is not fixed and there are more places on the panel. If you are interested or you know anybody who is interested in being and working on this panel, it would be very helpful. You can contact the 2 names listed at the bottom, Joanne Odenkirken and Phillip Yeskey, or any of us at NINDS, and we would be very grateful for even more input and people who are willing to work on developing the CDEs for mitochondrial disorders. Thank you for letting me ask that for the moment.

Dr Wallace: Thank you very much, Dr Hirtz. Now we would like to open up the discussion to you all who are the ones that are doing the hard work of seeing the patients, diagnosing the patients, trying to help them through this maze of different questions, answers, topics, tests, and then ultimately having to manage the patients, which is an area of tremendous importance in this disease process. One of the things in our experience is that relatively it's easier to do the diagnostic workup than it is to help that patient manage their life after you have a decision. You people do the really hard work of helping those people have as productive and happy life as possible. Do you have any questions for the panel?

Audience: I'd like to pick up on Dr Haas' and Dr Parikh's comments in particular about DNA testing, the issues of getting insurance approval, and then Dr Parikh's comment about segregation of mitochondrial wild versus affected in different body tissues. We have companies that advertise getting the buccal smear, getting a blood study, etcetera, and doing a DNA study without going into detail of what we're looking for. The question I'd like to ask is if we miss on say a buccal smear, does that mean we've lost an opportunity by not doing the study on muscle instead? Having lost that opportunity, can we go back to the insurance company and ask for another prior authorization to do a second study? I think we may only get one shot at this point in time. Thank you. 
Dr Haas: I think there are a couple of issues relating to genetic testing. The first is mitochondrial DNA sequencing and deletion analysis. The second of course is nuclear disease. Our nuclear gene's encoding mitochondrial proteins.

I don't think it was really brought up today, but in the pediatric age group, about $80 \%$ of disease is nuclear-encoded and $20 \%$ or less is mitochondrial DNA-related. In the adult age group, it's about $60 \%$ that's mitochondrial DNA-encoded.

There are 2 different things that we need to look for when we're doing gene testing. The tissue issue really only relates to mitochondrial DNA sequencing. Heteroplasmy typically is at its lowest level for most mitochondrial DNA diseases in blood and at its highest level in muscle. In between is saliva and renal epithelial cells.

Certainly if you have muscle available, that's the preferred tissue to look for mitochondrial DNA heteroplasmy. That will have the highest percentage of the pathogenic mutation in it.

The new next-gen sequencing methods are so sensitive that you can get down to a $1 \%$ level fairly easily of mutational load in any tissue. You will probably see the mutation in a patient's blood even if it's present at a very low level if it's a point mutation. That doesn't necessarily clinch the diagnosis, unless you find a higher percentage of mutation.

I think it is best to work with a muscle if you have it available, but certainly saliva is a good second choice. I think my third choice for mitochondrial DNA would be blood, with a rider that it really doesn't help very much for deletion analysis. In the adult age group, a lot of patients have mitochondrial DNA deletion disease. You really need muscle to detect that.

As far as the nuclear genes, which is a lot of the pediatric disease, any tissue you have will work fine. You're looking for genomic DNA. The insurance companies do just generally give you one shot at this. A number of the companies that are offering sequencing will provide a single code, which will do both mitochondrial DNA and nuclear DNA. I think that's often what I'm doing now. If the patient has good enough insurance to cover the testing, knowing that I may just get one shot at it, I'll ask for both things, even though I feel it's more likely to be a mitochondrial problem or a nuclear DNA problem. Sumit?

Dr Saneto: One caveat to that, at least in my experience, is that Pearson, KearnsSayre, CPO, there's a range of where you might find the deletion. The earlier disease more likely you're going to find it in blood. But if it's not expressed until later, you may miss it in blood and only pick it up in muscle. That just depends on your patient population, who you're sending testing on, and what you're thinking of. If you're really thinking that it may be CPO or late-onset Kearns-Sayre. You may really want to send muscle versus lymphocytes because you may miss it on lymphocytes.

Dr Parikh: As far as the insurance issue goes, I don't think I have an easy answer, except trying to explain the complexities of this to them.

I do want to kind of make a distinction. If we're talking about a problem in the nuclear DNA, obviously it's not an issue what tissue we have, whether it's a buccal swab, blood sample, or even muscle, although that would obviously be overkill. The distinction I think is 
much more so for mitochondrial DNA. Like Dr Haas was mentioning for mitochondrial DNA, point mutation analysis, genome sequencing, heteroplasmy detection level has improved to the point that at least, let's say for blood, for example, we can find low levels of heteroplasmy that often allows us to not need to go and get a muscle biopsy.

Buccal swab, I'm not quite sure. I even pose the question to some of the speakers in the audience. I don't know if anybody's looked at mitochondrial DNA heteroplasmy, level detection, or mutation burden in buccal swab. I fear at least theoretically we could potentially be missing diagnoses if we're just extracting mitochondrial DNA from buccal swabs.

Dr Parikh: Absolutely. Dr Hirano was just mentioning that they did look for heteroplasmy levels in MELAS in different tissues and found urine to be one of the best to help detect heteroplasmy levels that correlated a little bit better with deeper tissue levels. That's one issue, mitochondrial DNA point mutation analysis in buccal swabs. I don't know if that's enough.

Then where I think muscle and mitochondrial DNA analysis truly come together is deletion duplication analysis; that if you don't find an answer in blood, if you go looking for a deletion or duplication you truly may need muscle, as a variety of people have said.

Audience: Just another comment about the muscle biopsy. When we have a patient come in, they usually have very ill-defined symptoms of weakness, fatigue, encephalopathy, whatever it might be. We don't start with mitochondrial disease and work back. The value of the biopsy that we found is often there was a diagnosis that you didn't suspect. For example, we've had patients that have had nemaline rod myopathy and we didn't suspect it. They came in with hypotonia and a slight increase in CK and a little bit of increase in lactate. We thought it was mitochondrial, but when we got the EM back, we saw nemaline rods, made the diagnosis of an ACTA1 mutation.

I think that's another value of the muscle biopsy. You see things that you don't expect. It's particularly important in pediatrics to remember as well that the EM is very important. Often the late microscopic changes take time. They take time to actually manifest. Pleomorphic mitochondria and absence of cristae are sometimes the first manifestations, in addition to finding another disorder.

The other point I wanted to make about muscle biopsies is that in this era of next-generation sequencing we often have to go back. If you get a variant of uncertain significance in POLG, then you would ask the question, are there deletions or is there depletion in the muscle? If you don't have the tissue, then you have to go back. Sometimes it's helpful.

What we do is do the muscle biopsy late in electron microscopy on every child. At the time, we take a crest of skin that we use for fibroblast analysis and then we do the LP at the same time. Then we have the tissue so we don't have to go back and expose the child to anesthetic. When they're asleep, we get the consent and take a crest in a fibroblast that we can do our functional assays when we, for example, discover a new gene and we have to 
knock it down or play other games at the molecular level to prove it. That's another value in having muscle biopsy.

I've seen so many folks referred to me who had had mitochondrial sequencing done in blood and it's completely negative, even with very sensitive next-gen sequencing, things like cytochrome B mutations, for example, as one of the classic ones.

If you're going to go that route, I still think muscle is very important. I think I'm echoing what the panel is saying, but I wanted to bring forth the other value of the muscle biopsy.

Dr Wallace: I'd like to also reiterate that one of the reasons why muscle biopsies are a concern is because traditionally they require taking a fairly large piece of tissue. I think there are real opportunities now for reducing the amount of tissue and for refining our assays.

One of the things that we spent a fair amount on is building microfluidic chambers that can assay respiratory function on a volume of less than $100 \mu \mathrm{L}$. Then you only need 1000 cells. Then you can use the kind of needle biopsy that both Mark and Richard have used regularly without having a tremendous amount of invasive surgery done. I think there is a real opportunity to reinvigorate the muscle biopsy if we just change our methods.

Audience: I have a patient who I think might have mitochondrial disorder, do I send the blood for DNA testing? Then what kind of DNA testing? Mutation or deletion, duplication? What should be done first? If you go for muscle biopsy, when does that come into play? It adds up every little test. This is negative and you go to that and that.

Dr Goldstein: I think this is a very good question, probably one that I face with nearly every new patient that walks in the door. I think it's been said several times today that even if someone comes in with multisystemic symptoms, they don't necessarily have mitochondrial disease, so we have to use a very broad net initially, assuming there are other tests that have been done that might be more hypothesisdriven already, looking at other multisystemic diseases like the CDGs or even doing an oligo array, for instance. I think it depends on exactly what the symptoms are of the patient and whether it fits a known phenotype or not. It also depends on the family history. There are families that come in and I can readily pull out a maternal inheritance. When that's the case, then I want to go after mitochondrial DNA.

One of the questions that comes up a lot in my hospital is that there are several different types of mitochondrial DNA blood panels. There are these common mutations and deletions, which really only gives you MELAS, MERRF, NARP, and the Southern blot deletion for Kearns-Sayre or Pearson. Then you can do mitochondrial DNA sequencing. I only do the common mutations when I know what I'm looking at. If I know this is a family that probably has MELAS or MERRF or NARP or Kearns-Sayre, then I'll go after that first. Otherwise, I'll try to do the mitochondrial sequencing.

One of the issues with that—and I think we all have experience with this—is you get an abnormal result, but it's a rare homoplasmic variant and you don't know what it means 
because it hasn't been described before. Sarah Shansky warned me about this probably 15 years ago, that we're going to be in trouble when we start sequencing mitochondrial DNA, and she was right. However, if you look at the morbidity map right now of mitochondrial DNA, there are many more than 6 mutations and a deletion on there. We're getting better at knowing which mutations are pathogenic and which ones are going to be benign polymorphisms or related to your haplotype, where you come from in the world.

Again, I go to mitochondrial DNA if I have a strong suspicion of a maternal inheritance. Then I might do the nuclear mito panel for other kids.

Dr Haas mentioned that some of the labs are doing a combined panel. Some of the labs offer this dual massive parallel sequencing so you can do mitochondrial nuclear DNA at the same time. I am going after blood rather than tissue, at least initially, for children that I think have mitochondrial disease. Then I'll go back and explore whether I need to do a skin or muscle biopsy. That's drastically changed in the last 5 years for sure because of the availability of all the genetic tests that are now available to us commercially.

Dr Gropman: I agree in general. One has to consider other diagnoses, and sometimes we're fooled because the spectrum of congenital disorders of glycosylation can mimic or overlap mitochondrial symptoms. That has to be considered.

My first response when I heard the question is, "Well, what are the symptoms?" I think other than the family history and the careful clinical examination, are there other tests that have been done that can either bode for against mitochondrial disorders? What about neuroimaging? I find that there are some very strong patterns like you've seen today and others that will convince me more or less so that we're dealing with a mitochondrial disorder. What about the metabolism pattern? Often we're checking labs on children who are at baseline. The opportunity to study organic acids, amino acids, carnitine, ACL carnitine, lactate, etcetera. When they're ill, it is also important in trying to determine if we're dealing with a mitochondrial disorder.

I just wanted to make one comment about the biopsy. I think that if you're going to do a biopsy, you really have to think about what type of information you want to get from the biopsy. You're only going to have really one chance in a child to put them under anesthesia and get what you need to get. I think you can't really take that lightly.

The other thing that we've done at my hospital is to take advantage of other sedated procedures that may be occurring, whether it's an LP or the child is going in for a surgery to either get muscle or skin at that time so that we limit the exposure to anesthesia.

Dr Haas: I would just add one thing. We haven't really talked about it, but anesthesia carries a significantly increased risk for mitochondrial disease patients. That needs to be part of the calculation. I entirely agree with Andrea that if they're going to have a surgical procedure anyway, that's the time to get the muscle biopsy. 
Audience: I have a patient who has congenital HIV infection who is on several antiretroviral medications. He does have seizures. He's a little bit encephalopathic. Would I suspect secondary mitochondrial disorder dysfunction? How would I work him up? What should I do?

Dr Goldstein: The antiretroviral drugs are known to be mitochondrial poisons. A lot of the AIDS-related secondary side effects from the meds are known to be because of the lipodystrophy, for instance. I think there have been papers published already about putting patients on these medications on a mito cocktail per se-carnitine and CoQ10 among others — and there's been improvement. Anyone, add to that, please.

Dr Gropman: Most of the research is on adults with HIV who have been on these agents and the HIV lipodystrophy syndrome with elevated lactate. There have been several publications over the last few years that address this. Many of the patients have benefited from treatment that you would apply to a patient who has a primary mitochondrial disorder.

The other population of patients studied are the offspring of women who have HIV who are exposed to these drugs, and their children are exposed in utero. The consensus on that is not as clear. Audience: The same goes for antipsychotic medications. You have children with autism and they're on Risperdal forever. What do you do with them?

Dr Haas: The way that I work up patients with a question of mitochondrial autism kind of matches your HIV question. We order a collection of biochemical tests. I would routinely look at the lactate level without a tourniquet, CPK, plasma amino acids, looking for changes in the alanine, ACL carnitine profiles, looking for evidence of mitochondrial dysfunction that affects fatty acid oxidation, and organic acids.

That's kind of a routine when we're casting the net wide looking for mitochondrial disease. That certainly fits well when you're wondering if a patient has a drug-induced mitochondrial problem. I guess the additional thing about the retroviral inhibitors is that they tend to cause hepatic disease as well. You certainly want to make sure you're looking at liver enzymes also.

Audience: My question is about clinical relevant endpoints. For future trials, as we mentioned, we need clinically relevant endpoints. How can we establish these endpoints in these diseases, which are rare and there's a greater clinical variability?

Dr Goldstein: We are in the process of forming committees to work on these clinically relevant endpoints. Because mitochondrial disease is so heterogeneous, we know that this is going to have be somewhat disease-specific. For instance, if we want to come up with clinically relevant endpoints for Leber's hereditary optic neuropathy, that's going to have a lot to do with visual acuity, color vision, and so on. For Kearns-Sayre, they're going to be totally different endpoints. We anticipate that this is going to be an enormous task.

Again, if anyone wants to help out on those committees, please email me or Phil Yeskey. We actively want to have as many people participating and also have it be an international collaboration. Again, the points is that if somebody is doing a clinical trial for Leber's, that 
one group isn't looking at just visual acuity and we have another group looking at just color vision, because we want to be able to compare those results.

Dr Saneto: We really need to learn the natural history of a lot of these disorders that we really don't understand. I thought I knew a lot about Alpers. But every time I see another Alpers kid, I learn something new. Same mutation; just different presentation. Their course is a little bit different. If we knew a little bit more about the natural history, we might be able to intervene. If we don't know the natural history, we're really not sure what that intervention is really accomplishing in total. It's just another plug for NAMDC. We need to learn a lot more.

Dr Maria: When thinking about the published proceedings in NDC the last 13 years, by far the most accessioned paper by an order of 4:1 over everything else has been the consensus statement that Dr Chin Wang published on spinal muscular atrophy, an incredibly used set of guidelines. I guess we've heard that as a challenge or an opportunity that there's a lot of variability in management.

What would it take to build consensus in the community and to develop a set of guidelines? When I think about how far advanced SMA was, in many ways we know more about mitochondrial than we knew of SMA at the time of that consensus statement, and yet it served a real purpose of harmonizing at least the clinical community to a set of practices. Why don't we have such a consensus statement already?

Dr Parikh: The Mitochondrial Medicine Society is working on exactly that. There's already a committee that's formed. All their subgroups have their assignments. People are researching their topics. The goal is, if we can glue all of this together, to come up with a working document sometime next year that we can offer as guidance until we get new knowledge and maybe rewrite those. There's definitely something in the works and planned.

I think our first step was to focus on diagnostic methods used and a little bit on supplement therapy, but we also want to look at how to follow and track these patients long term. How many echocardiograms do you need to do? How many EKGs do you need to do and how often? There have already been groups abroad that have been working on this and we also need to make sure that we collaborate with them. Partly not to re-create the work if it's already been done very well, but partly because I think our patient populations will vary and our approaches may vary as well.

Dr Maria: Will you all be using the same sort of Delphi criteria methodology that was used by Dr Wang and colleagues in approaching SMA?

Dr Parikh: Yes, along with the Oxford Levels of Evidence guide. Audience: Many labs offer gene testing. Do you have-I don't want to use the word "favorite"- but maybe trusted labs that you send to? What's the shortest time that it takes DNA sequencing test to come back? I was told in one lab 12 weeks and I felt that's a little bit long. Thank you.

Dr Haas: Let me just say one thing about this. We've seen that the cost of doing sequencing is dropping dramatically, but I don't know if the rest of you have noticed that the pricing for having this testing done really hasn't changed much 
over the last couple of years. That's because the major issue here is bioinformatics. It's interpretation of the results and doing a lot of behind-the-stage research to produce the report. I think that is what is largely taking a lot of the time in getting these results back. I don't know how much can be compressed because it's dependent upon a lot of humans working as hard as they can.

Dr Gropman: That's absolutely right. The technology to do the sequencing is fairly straightforward and quick, but the hard part is how do you interpret the mass of information? Depending on the laboratory, there are multiple levels of confirmation of the results that come back.

I found it a useful experience for one of our rotating fellows to actually go to one of these DNA diagnostic facilities as a field trip. She spent a whole day there. She followed a sample through from the barcoding to the sequencing.

Basically, after she came out of that experience, she said, "Wow. Now I know why it takes so long to get the sample back." It's true. The actual technology is not what is holding it up, but it's the interpretation and the bioinformatics.

Dr Parikh: I'll add one more thing to that. The question of what are our favorites, I don't think any of us are going to necessarily answer that. I can kind of give you a general overlay of the land. I posted this on the child neurology listserve as well. We have more options now than we ever did. Next-gen panels that are available, there are some disease-specific panels. If you suspect a mitochondrial depletion disorder, you could get these 20 genes.

There are larger gene panels, like 100-plus gene panel, a 400-plus gene panel, and then there is somebody who's offering I believe around 12 to 14,000 genes as well. I think a lot of that is going to end up being one personal preference as to which lab they choose to what are you exactly looking for? Third, does the lab offer deletion duplication analysis along with point mutation analysis? I think that's important. I'm losing count. I think I'm on 4. How are the reports written? How are variants interpreted? Is everything flagged? Do you get a 3-page report that's weighing everything equally or are you getting something that helps you sift through what's important and what's not?

I think all of that has to be taken into account and I don't know if there truly is just one lab that everybody should be using all the time. You might need to be able to pick and choose. Be aware of what's available and then pick and choose depending on your clinical situation.

Dr Gropman: I would agree that cost and coverage are important, but also customer service. Some of the labs that I prefer may not necessarily be the ones that do the most extensive panel, and sometimes I don't need that, but can I call up and speak to somebody? How much of the interpretation work have they done and how much have they left for the clinician to really figure out on his or her own is important to me as well.

Audience: I have a question about infantile spasms. Dr Saneto mentioned his percentage of patients with mitochondrial and infantile spasms. We routinely go through all the testing that's been mentioned: lactate imaging. When then do you 
push beyond all of those being normal, going from mitochondrial analysis and infantile spasms? Common problem and the end result is as you said. ACTH, steroids, maybe prednisone. Where do you go next?

Dr Saneto: I think it varies. Some kids kind of present with Leigh's syndrome and that's sort of a pretty easy diagnosis. It's the ones that are different. It's only recently that we've been picking up some. We have a couple that are MTOIs. We have a couple that are some other single gene mutations that have been only described a few times.

It's usually during the aftermath. Pretty much, I can control things- this is no advertisement -with ACTH. Sometimes I need the combination or the ketogenic diet and vigabatrin together, and I can make the spasms go away and the EEG normalizes. Then most of my patients end up with intractable seizures after that. That's when I start rethinking. What I was thinking would be cytogenic, now I'm thinking idiopathic. Based on what I see on the EEG and the other organ involvements, then I start walking down the mitochondrial pathway.

Audience: Do you do muscle biopsies on these kids with seizures?

Russ Saneto: Usually what happens is I revisit the MRI scan. During the anesthesia for the MRI scan I'll get a muscle biopsy at that point in this little piece of skin now that I'm trying to do. Usually I couple it with another procedure so I don't have to do too many anesthesias.

Dr Parikh: I would posit that most of the patients with infantile spasms that we see that have unremarkable MRIs, even though we may go fishing for a mitochondrial problem by looking at these biomarkers, the vast majority are going to have a mutation in one of the epileptic encephalopathy genes. In my patients if there is nothing else with a big arrow saying that this is mitochondrial, I usually have been running to those gene panels to look for a problem there.

Then when that's negative, the other place I've been looking is spinal fluid. Because we know that there are other patients with treatable metabolic disorders that sometimes the gene panels don't always pick up and we definitely don't want to miss those. I think that's kind of our stepwise approach. Then I would say third, if we're still without a diagnosis, is going back and thinking could this be an atypical presentation of a mitochondrial disorder. This is again on the flip side with a completely normal MRI and nothing clearly indicating this could be mitochondrial.

Audience: I just want to make one very quick comment in response to not the previous question, but 2 before about which exome sequencing panel might be good. By no means am I going to endorse a particular company or university or laboratory at this point, but I will make a plug for trio sequencing for the parents and the child. It helps much. The bulk of the cost of these tests is the interpretation, as Richard pointed out. Many of these companies and universities will actually just for a little bit more money simultaneously sequence the parents. That helps big time in the interpretation. That allows you to phase the haplotype of the variants. If you're looking for a 2-hit gene, that's next to impossible to do with short reads 
that's the current state-of-the-art. It allows you to haplotype the variants and then determines which ones are compound heterozygous.

On top of that, most people, if you have a singleton case of disease, you can only interpret the recessive variants. If there is a de novo mutation, that's actually something that you can discover, a dominant de novo, either dominant that's de novo or it allows you to exclude the possibility of other heterozygote experiences. It's a huge, huge boon. It doesn't cost that much more and I think it helps in the interpretation.

Dr Schon: Normally I would make a comment that's global and philosophical, but I'm going to be quite the opposite and be highly technical. The comment that Dr Gropman made that the whole genome sequencing is easy, is correct. But it has the potential to be fundamentally flawed. I just want to talk about it for a minute because you are the people who are going to be actually sending guidelines.

The issue is that the nuances of sequencing whole genome - and Vamsi, maybe you can help me out on this - is that you can amplify mitochondrial DNA pseudogenes that could be misinterpreted as being variance, mutations, and who knows what. That's because total cellular DNA is the template for the whole genome sequence. You certainly cannot find the deletions.

I would urge that if there were a consensus statement on diagnosis, that that consensus would say when you're looking for nuclear mutations, go ahead. Be my guest; do it. But if you want to look at mitochondrial DNA mutations, spin out the nuclei, isolate the mitos, do something to get rid of that possibility. I think it's a real danger point. If we're going to do real mitochondrial DNA diagnosis, let's look at real mitochondria.

Douglas Wallace: Thank you, Eric. I'd like to then close this session by very much thanking the panel members for their excellent insight, for all of you for being here, and for the people that have made questions and comments. I'd also like to take this opportunity to thank Bernard Maria, who's been instrumental in contacting many of us and really taking this vision to its conclusion and very successfully through his program in Neurobiology of Disease in Children.

Dr Maria: My closing comment is simply that we take great pride in finishing ahead of schedule every year. We're going to do that now. I do very much appreciate the leadership of our superb codirectors. I appreciate all of you and your support and the fact that here we are at the end of the day - a heavy day, a lot of exciting information - and you all are still here. You obviously have exceptional mitochondrial metabolism and stamina. I look forward to seeing you in Columbus when we tackle autism at NDC 2014. Thank you all again.

\title{
Acknowledgments
}

\author{
Funding \\ The authors received no financial support for the research, authorship, and/or publication of this article.
}




\section{References}

1. Minczuk M, Papworth MA, Miller JC, et al. Development of a single-chain, quasi-dimeric zincfinger nuclease for the selective degradation of mutated human mitochondrial DNA. Nucleic Acids Res. 2008; 36:3926-3938. [PubMed: 18511461]

2. Brown DT, Samuels DC, Michael EM, et al. Random genetic drift determines the level of mutant mtDNA in human primary oocytes. Am J Hum Genet. 2001; 68:533-536. [PubMed: 11133360]

3. Bacman SR, Williams SL, Pinto M, et al. Specific elimination of mutant mitochondrial genomes in patient-derived cells by mitoTA-LENS. Nat Med. 2013; 19:1111-1115. [PubMed: 23913125]

4. Jeppesen TD, Schwartz M, Olsen DB, et al. Aerobic training is safe and improves exercise capacity in patients with mitochondrial myopathy. Brain. 2006; 129:3402-3412. [PubMed: 16815877]

5. Taivassalo T, Gardner JL, Taylor RW, et al. Endurance training and detraining in mitochondrial myopathies due to single large-scale mtDNA deletions. Brain. 2006; 129:3391-3401. [PubMed: $17085458]$ 